\title{
Uncertainty Quantification in Molecular Dynamics Studies of the Glass Transition Temperature ${ }^{\text {th }}$
}

\author{
Paul N. Patrone ${ }^{\mathrm{a}, \mathrm{b}, *}$, Andrew Dienstfrey ${ }^{\mathrm{b}}$, Andrea R. Browning ${ }^{\mathrm{c}}$, Samuel Tucker ${ }^{\mathrm{c}}$, Stephen Christensen ${ }^{\mathrm{c}}$ \\ ${ }^{a}$ Institute for Mathematics and its Applications, University of Minnesota \\ ${ }^{b}$ National Institute of Standards and Technology \\ ${ }^{c}$ The Boeing Company
}

\begin{abstract}
The composites industry is increasingly using molecular dynamics (MD) simulations to inform its materials development decisions. As a result, there is growing awareness that simulated predictions require quantitative assessments of their quality in order to routinely provide reliable and actionable information. In the following, we develop a suite of uncertainty quantification (UQ) tools designed to assess simulation-based estimates of the glass transition temperature $T_{\mathrm{g}}$ of polymer systems for aerospace applications. We consider contributions to this uncertainty arising from: (i) identification of asymptotic regimes in density versus temperature relations; (ii) fluctuations associated with limited time-averaging of dynamical noise; (iii) and finite-size effects associated with partial averaging over polymer-network configurations. We present a sequence of analyses by which we assess each of these contributions and quantify their net effect on estimates of $T_{\mathrm{g}}$. Importantly, these methods suggest more efficient workflows by indicating when multiple small simulations can be combined to yield estimates with uncertainties comparable to larger, more expensive simulations. We expect that related approaches will, in the future, be applicable to other physical quantities of interest as well as to a broader class of computational tools.
\end{abstract}

Keywords: Uncertainty quantification, Glass transition temperature, Crosslinked polymers, Molecular dynamics, High-throughput materials-modeling workflow

\section{Introduction}

With the growing power and availability of high-performance computers, the composites industry is investing in molecular ${ }_{26}^{25}$ dynamics (MD) as a cost-effective tool for identifying next generation materials [1-3]. In such applications, MD is used to rapidly screen a combinatorially-large design space for polymer systems whose mechanical properties warrant further experimental study. While this strategy saves time and money, computational limitations can lead to situations that require a balance between accuracy and precision on the one hand, and high throughput on the other. Thus, there is a need for analytical techniques to assess confidence in MD simulations, thereby ${ }_{34}$ rendering their predictions more informative for decision mak- ${ }_{35}$ ing in the materials development cycle. The growing field of uncertainty quantification (UQ) provides a foundation from 36 which to develop and apply these techniques $[4,5]$.

Simulation-based prediction of the glass transition temper- 38 ature $T_{\mathrm{g}}$ is an especially relevant and challenging problem ${ }_{39}$ where UQ tools are needed. In composites, $T_{\mathrm{g}}$ characterizes the boundary between rigid and fluid-like behavior of the con- 40 stituent polymers. At the bulk scale this translates to the abil- ${ }^{41}$ ity of a structural component (e.g., an airplane wing) to remain ${ }^{42}$

\footnotetext{
This work is a contribution of the National Institute of Standards and Technology and is not subject to copyright in the United States.

${ }^{*}$ Corresponding author

Email address: paul patrone@nist.gov (Paul N. Patrone )
}

stiff at elevated temperature [6]. Despite being both a natural and critical property of polymers, computing $T_{\mathrm{g}}$ from MD simulations presents several challenges. For one, the dynamic processes associated with glass formation are inherently nonequilibrium. Moreover, the underlying molecular structure of crosslinked polymers is a random network. Thus, in order to sufficiently average cross-linking statistics and collective dynamics, it is desirable to simulate macroscopic systems over long times. Given that MD falls short of these scales by several orders of magnitude, modelers often face the following question:

How trustworthy are estimates of $T_{\mathrm{g}}$ obtained from molecular dynamics simulations?

Importantly, the same question applies to predictions of any bulk material property obtained from atomistic simulation.

In this paper we develop a number of tools to address this question. Specifically, we consider:

(I) a hyperbola-based regression model that (a) assesses whether a simulated density-versus-temperature dataset is consistent with the definition of $T_{\mathrm{g}}$ and (b) if so, estimates $T_{\mathrm{g}}$;

(II) a pooling analysis in which we compare groups of individual datasets to assess convergence of the simulations as a function of simulation size $N$; 
(III) a noise-propagation model that assigns uncertainties to 99 $T_{\mathrm{g}}$ values derived from the hyperbola analysis of individ-100 ual datasets;

IV) a weighted-mean statistic that (a) quantifies dispersion be- ${ }^{102}$ tween datasets and (b) yields a consensus value of $T_{\mathrm{g}}$ by ${ }_{104}$ weighting the estimates from all datasets according to the ${ }_{105}$ aforementioned uncertainties. ${ }^{1}$

We present this analysis as an example of a systematic work- ${ }^{107}$ flow leading from generation of data to its final interpretation. ${ }^{10}$

A primary theme motivating this strategy is the need for ${ }^{109}$ workflows that support objective, reproducible, and automated ${ }^{110}$ predictive capabilities. Consider, for example, that simu- ${ }^{111}$ lated estimates of $T_{\mathrm{g}}$ are usually extrapolated from subjectively ${ }^{112}$ identified, asymptotic regimes of density-vs-temperature data; ${ }^{113}$ cf. Fig. 1. The ad hoc nature of this procedure can lead to di- ${ }^{114}$ vergent results-one analyst may identify asymptotic regimes ${ }^{115}$ resulting in a $T_{\mathrm{g}}$ estimate with an unfounded confidence, while ${ }^{116}$ another might conclude that the data have not asymptoted $a t^{177}$ all. Our analysis circumvents this problem by fitting all points ${ }^{118}$ in a dataset to a hyperbola regression model. This approach ${ }^{119}$ eliminates case-by-case identification of asymptotic regimes, ${ }^{120}$ reduces the need for input by the modeler, and increases re- ${ }^{121}$ producibility of the $T_{\mathrm{g}}$ estimates. Combining this regression ${ }^{122}$ with a noise propagation model also quantifies the uncertainty ${ }^{123}$ of our estimates in light of dynamical fluctuations in the sim- ${ }^{124}$ ulated data. Such indicators of robustness are essential for in- ${ }^{125}$ formed decision making.

A second, related theme stems from the need to investi- ${ }^{127}$ gate consistency between atomistic simulation and desired bulk ${ }^{128}$ scales. Given that direct simulations of macroscopic systems ${ }^{129}$ are not possible, we instead consider the extent to which our ${ }^{130}$ hyperbola-based estimates have converged as a function of sys- ${ }^{131}$ tem size. In this context, our pooling analysis averages collec- ${ }^{132}$ tions of small-scale simulation results as proxies for increas- ${ }^{133}$ ingly large computations. Importantly, such procedures can re- ${ }^{134}$ veal the presence of bias in the $T_{\mathrm{g}}$ estimates, which arises from ${ }^{135}$ a non-linear transformation of noise in a dataset as it is propa- ${ }^{136}$ gated through an analysis. Moreover, the pooling analysis can ${ }^{137}$ indicate when and by roughly how much the system size should ${ }^{138}$ be increased to control this bias.

Finally, we emphasize that multiple steps in the simulation ${ }^{140}$ workflow contribute to non-trivial uncertainties, all of which ${ }^{141}$ must be jointly modeled to account for the total uncertainty as- ${ }^{142}$ sociated with an estimate of $T_{\mathrm{g}}$. In particular, we show that un- ${ }^{143}$ certainty estimates based only on noise propagation applied to a single nanoscale simulation may result in predictions that are $_{144}$ overly optimistic, if not statistically inconsistent. We attribute this to the prospect that noise within a given dataset arises from ${ }_{145}$ dynamical fluctuations based on a single instance of the cross- ${ }_{146}$ linking statistics and limited exploration of the energy land- ${ }_{147}$ scape. As such, these fluctuations may not capture the variability between datasets, the latter corresponding to the differences ${ }_{148}$

${ }^{1}$ In a weighted-mean statistic, each simulation contributes unequally to the $\mathrm{e}^{150}$ average $T_{\mathrm{g}}$ value. in independent samples taken from a bulk material. Borrowing analysis from the metrology community, we estimate this additional between-simulation uncertainty using a mixed-effects statistical model. The scale of this so-called "dark uncertainty" in relation to the within-uncertainties derived from individual datasets may be viewed as another indicator by which to ascribe confidence to the simulation-based experiment.

We briefly define the scope of this work. We do not precisely identify the origins (physical or otherwise) of uncertainty in the course of our analysis. Generally speaking, we implicate fluctuations associated with statistical mechanics, although this neglects effects such as model-form error, etc. The task of identifying specific contributors to the uncertainty requires that one untangle their potentially complex interdependencies, which lies beyond the present study. Similarly, we do not address questions related to modeling inter-atomic force-fields. References [7-11] consider calibration of parameterized force fields and propagation of the associated uncertainties into subsequent computations for ionic systems. We speculate that a related analysis for polymers could be appended to our workflow and summarized in terms of a between-model uncertainty akin to that in step IV above; such studies are left for future work. Finally, we omit comparison of computational results with experiment. In UQ terminology, our focus is on verification, broadly defined as an analysis of uncertainty contributions arising entirely from within the computational domain. Following common practice, these sources of uncertainty are analyzed prior to comparison with experiment, or validation, so as to sharpen the inferences resulting from the latter.

The rest of the paper is organized as follows. In Section 2, we review a common definition of the glass transition temperature and provide an overview of our MD simulation protocols. In Section 3, we develop the non-linear regression model by which we estimate $T_{\mathrm{g}}$ from simulation data. In Section 4 we derive a criterion to assess the system-size dependence of our regression analysis and show how a pooling analysis can be used to verify this convergence. In Section 5 we introduce a mixed-effects statistical model, develop the necessary tools to estimate withinand between-simulation uncertainties, and derive a final consensus estimate of $T_{\mathrm{g}}$ that takes into account results of all of the aforementioned uncertainties. We conclude with a discussion of past works, limitations, and extensions. Appendices provide relevant information on convergence and equilibration of simulations, as well as certain mathematical details underlying our analyses.

\section{Background}

In this section, we provide background on measurement and simulation of the glass transition temperature, highlighting issues relevant for UQ.

\subsection{The glass transition}

Informally, $T_{\mathrm{g}}$ is the temperature above which a polymer system becomes soft. Experimentally, this transition is determined by a variety of procedures that are distinguished by refinements 
this notion [6]. For comparisons between experiments and simulations, it is common to infer $T_{\mathrm{g}}$ from the density-versustemperature curve, $\rho(T)$. Experimentally, the density of a sample is measured as the system is annealed at a fixed rate on the order of $10 \mathrm{~K}$ per minute. In silico we reproduce this procedure, albeit at significantly faster cooling rates (e.g., $\sim 10^{9} \mathrm{~K} / \mathrm{min}$ ), due to the timescale limitations of MD. ${ }^{2}$

The estimation of $T_{\mathrm{g}}$ from $\rho(T)$ data is based in the observation that, asymptotically, density varies linearly with temperature when the material is in its glassy or rubber-like states, i.e., at the extremes of low and high temperatures respectively. The glass transition itself is associated with a rapid change in the slope of $\rho(T)$ bridging these two asymptotic regimes. Convention returns $T_{\mathrm{g}}$ as the intersection of two best-fit lines extrapolated from the low and high temperature data; see Fig. 1 and Refs. [12-22]. In experiments, this procedure is generally acceptable given that the transition region is relatively sharp and visibly confined to an interval of a few tens of degrees or less. In MD simulations, however, the transition is less sharp and can occur over several hundred degrees; see Fig. 1 and Ref. [22]. Moreover, simulated densities are typically defined as a fixed mass divided by dynamically averaged volumes. As such, they exhibit finite-size effects that appear as noise, especially at high temperatures; see also Fig. 3 in Ref. [16]. Both of these features confound attempts to estimate $T_{\mathrm{g}}$ from a simulated annealing curve, and are characterized by the "within-uncertainty" analysis described below.

\subsection{Simulation methods}

Our simulation results are generated using custom scripts in Materials Studio [23]. ${ }^{3}$ Interatomic forces are computed using the COMPASS II forcefield, while temperature is controlled with the Andersen thermostat [24]. The simulation proceeds ${ }^{202}$ in two stages: (i) a reactive, curing stage in which a branched ${ }^{203}$ polymer system is built from monomer constituents; and (ii), $\mathrm{a}^{204}$ non-reactive, annealing process in which the system is cooled ${ }^{205}$ in step-wise temperature increments. We briefly describe these stages here; see also Refs. [3, 15, 16] for related methods.

The curing stage begins with a periodic cell of unreacted monomers. Taking this as input, the script iterates be- ${ }^{209}$ tween short dynamics simulations and nearest-neighbor bond- ${ }^{210}$ ing steps. Pairwise distances between reactive sites are com- ${ }^{21}$ puted, and all possible bonds within a prescribed distance are formed. The newly bonded system is integrated forward in time ${ }^{21}$ to relax the artificial stresses created. If, after this relaxation, ${ }^{214}$ additional reactive pairs are found within the same cutoff, the ${ }^{215}$

\footnotetext{
${ }^{2}$ We note that experimental measurements of $T_{\mathrm{g}}$ can be determined from ${ }^{218}$ changes in other mechanical properties such as modulus of elasticity. The pre-219 cise definition of $T_{g}$ should be explicitly taken into account during validation 220 of simulated results. Similarly, we do not consider the shift of $T_{\mathrm{g}}$ due to the significantly faster cooling rate in MD. Again, our focus here is on verification.

${ }^{3}$ Certain commercial equipment, instruments, or materials are identified in ${ }^{22}$ this paper in order to specify the experimental procedure adequately. Such identification is not intended to imply recommendation or endorsement by the National Institute of Standards and Technology, nor is it intended to imply that the materials or equipment identified are necessarily the best available for the purpose.
}

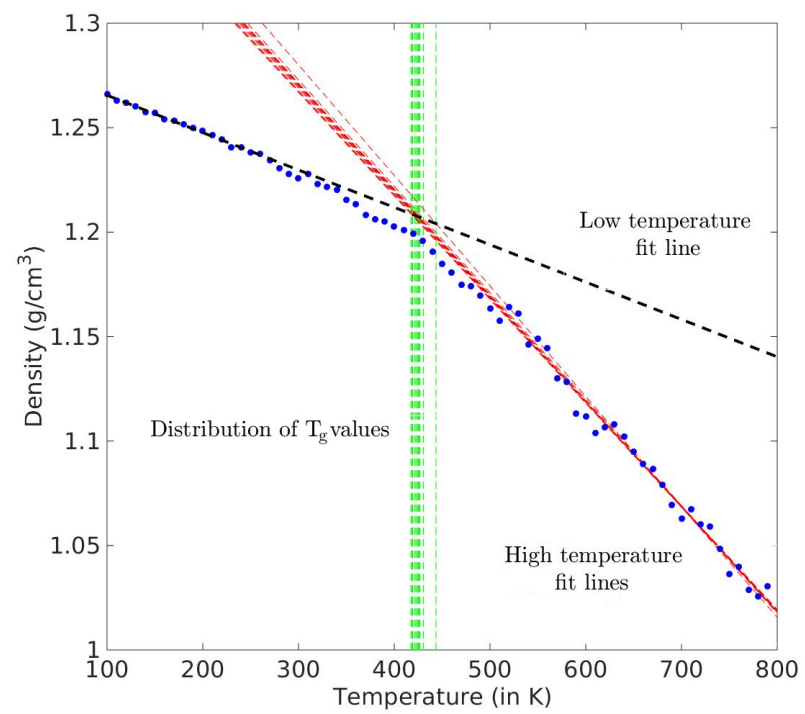

Figure 1: Multiple estimates of $T_{\mathrm{g}}$ from a single simulation. $T_{\mathrm{g}}$ (vertical green) is estimated as the intersection of low (dashed black) and high (dashed red) temperature best-fit lines, which in principle describe asymptotic behaviors of the data. Here we computed the set of high-temperature lines by leaving out each data point in turn from the $650 \mathrm{~K}$ to $800 \mathrm{~K}$ domain and fitting the remaining 14 points. The dispersion in $T_{\mathrm{g}}$ suggests that there are many valid interpretations of this data. (This dataset was generated by a 3800 atom simulation of 33BF; see Sec. 2.2 for discussion of the chemistry.) temperature $(350 \mathrm{~K}$ or 725 $\mathrm{K})$, determines the final crosslink percentage. ${ }^{4}$ For our systems, the final crosslink percentages fall between about $65 \%$ to $95 \%$.

We employ two barostat protocols to control the pressure during the reactive stage. Up to $50 \%$ crosslink percentage and after each bonding step, we relax the system using the Andersen and Parrinello barostats sequentially for 5 ps each [24, 25]. This ensures that the unit cells do not become overly skewed while the monomers are unconstrained and mobile. Above 50\%, we exclusively use the Parrinello barostat (10 ps after each bonding step) to relax internal stresses. Variation in the network structure of each realization is achieved by assigning random velocities to the initial box of monomers and then rerunning the curing simulation. We interpret each of these realizations as a small sample drawn at random from the bulk. As we show, a significant source of uncertainty in $T_{\mathrm{g}}$ comes from the variety of structures that are created during crosslinking.

Once cured, the system is heated to $800 \mathrm{~K}$ and cooled to 100 $\mathrm{K}$ in $10 \mathrm{~K}$ increments. With each increment the system is allowed to equilibrate to the new temperature for a minimum of 20 ps. Once equilibrated at a given temperature, the script runs additional simulations in order to compute an average density. The number of time-steps used for both equilibration and aver-

${ }^{4}$ The crosslink percentage is defined as the total number of bonds formed divided by the total number that could form. This latter quantity is determined from the stoichiometry of the system and number of monomers. 
aging is decided on-the-fly by a convergence criterion that re-276 quires fluctuations in the running-average density fall below a 277 user-defined threshold. Thus, the run-times of simulations dif-278 fer slightly from one to the next; in practice, variations are only ${ }_{279}$ on the order of a few percent. See Appendix A for a detailed 280 description of the convergence criterion and issues associated ${ }_{281}$ with equilibrating our systems.

In order to generate the data presented in this work, we ran ${ }_{283}$ the the curing and annealing scripts multiple times for each 284 chemistry. Post-simulation analysis of the data was done in $_{285}$ Matlab [26].

\subsection{Polymer systems}

The difficulty in simulating and analyzing polymer systems is partly determined by their monomer functionality, i.e., the ${ }^{289}$ number of possible bonding sites of the molecular constituents. We consider two representative amine-cured epoxies to illustrate different possible outcomes of our simulation workflow.

Our first system, denoted 33BF, is composed of: 3,3diaminodiphenyl sulfone (33DDS), a four-functional amine; and digycidyl ether of Bisphenyl $\mathrm{F}$ (BisF), a two-functional epoxy. 33BF is relatively easy to model; the simulations yield annealing data with small dynamical fluctuations and corre-290 spondingly consistent $T_{\mathrm{g}}$ values. We simulate $33 \mathrm{BF}$ systems ${ }^{291}$ having approximately 2400 atoms, 3800 atoms, 7600 atoms, ${ }^{292}$ and 16500 atoms.

The second system that we model, denoted 3344MY, is com- ${ }^{294}$ posed of: 33DDS; 4,4-diaminodiphenyl sulfone (44DDS); and ${ }^{295}$ tetraglycidyl methylene dianiline (MY720), a four-functional ${ }^{296}$ epoxy. 3344MY is representative of polymer systems that are ${ }^{297}$ difficult to model. Relative to 33BF, 3344MY can form a wider ${ }^{298}$ variety of crosslinked structures, and we speculate that the en- ${ }^{299}$ ergy landscape is more complicated. Operationally, this mani- ${ }^{300}$ fests as larger density fluctuations in the $\rho(T)$ curves, since ran- ${ }^{301}$ dom sampling of a given system size explores a smaller frac- ${ }^{302}$ tion of the total possible configurations. As a result, asymptotic ${ }^{303}$ regimes can be harder to identify, and the corresponding transi- ${ }^{304}$ tion region can appear smooth and occupy a large portion of the ${ }^{305}$ computational temperature window. For this system, we simu- ${ }^{306}$ late unit cells having roughly 4400 atoms, which are comparible in size to the $380033 \mathrm{BF}$ systems.

\section{Global hyperbola fit}

Conventional estimates of the glass transition temperature are imprecise for multiple reasons. For one, finite-size and -307 time effects manifest as noise that hinders the identification of 308 asymptotic temperature regimes defined by linear trends. More-309 over, $T_{\mathrm{g}}$ estimates based on extrapolation from temperature ex-310 tremes can be overly sensitive to noise, even if the asymptotic ${ }^{311}$ regimes are confidently chosen. Figure 1 illustrates these prob-312 lems for typical simulation data. We subjectively define the "high-temperature" regime as $650 \mathrm{~K} \leq T \leq 800 \mathrm{~K}$, although the fit lines suggest that temperatures down to $500 \mathrm{~K}$ could serve as a plausible lower bound for this dataset. Once this asymptotic region is identified, we fit lines to multiple subsets of the data in the high temperature regime as a proxy for sampling the effects of noise. As shown in Fig. 1, even though local differences in these fits may be minor for $600 \mathrm{~K} \lesssim T \lesssim 800 \mathrm{~K}$, extrapolation to the intersection with the low-temperature asymptote amplifies the uncertainty in $T_{\mathrm{g}}$.

In this section we present an alternative method for estimating $T_{\mathrm{g}}$ based on a hyperbola model for $\rho(T)$. Our approach is global in that it makes use of all simulated data simultaneously. This dispenses the problem of subjectively identifying asymptotic regions. ${ }^{5}$ What is more, the residuals - differences between the fit and data - provide a natural scale for estimating the uncertainty of the fit. We expand upon this idea in developing the noise propagation analysis of Sec. 5.1.

\subsection{Hyperbola-fit calculation of $T_{\mathrm{g}}$}

A fully-general equation for a single-branch of a hyperbola in the $\rho, T$-plane can be expressed via the formula

$$
\begin{array}{r}
\rho(T)=\rho_{0}-a\left(T-T_{0}\right)-b \mathcal{H}_{0}\left(T, T_{0}, c\right), \\
\mathcal{H}_{0}\left(T, T_{0}, c\right)=\frac{1}{2}\left(T-T_{0}\right)+\sqrt{\frac{\left(T-T_{0}\right)^{2}}{4}+\mathrm{e}^{c},}
\end{array}
$$

where $T_{0}, \rho_{0}, a, b$, and $c$ are constant parameters to be determined [29]. Geometrically, the role of these parameters can be understood as follows. First, in the limit that $c \rightarrow-\infty$, the function $\mathcal{H}_{0}$ approaches a piecewise linear function that is identically zero for $T<T_{0}$ and has unit slope for $T>T_{0}$; see Fig. 2. The parameter $c$ smooths out the discontinuity in the slope at $T=T_{0}$, and thus controls the transition between asymptotes. The point $\left(T_{0}, \rho_{0}\right)$ is the hyperbola center. The parameters $-a$ and $-(a+b)$ are the slopes of the asymptotic low and high-temperature regimes, respectively. For our applications, we may assume that $\rho_{0}$ and $T_{0}$ are both positive, since these correspond to densities and temperatures. Likewise, we expect that $a$ and $b$ are positive, as density is a concave function of temperature for polymer systems. The parameter $c$ may take any value $-\infty<c<\infty$. We denote the collection of these parameters generically as a vector $\phi$, and the resulting density as a function of temperature by $\rho_{\mathcal{H}}(\phi, T)$.

Within a fixed annealing run we denote the vector of simulated temperatures by $\mathbf{T}=\left(T_{1}, T_{2}, \ldots T_{D}\right)$ and $\boldsymbol{\rho}=\left(\rho_{1}, \rho_{2}, \ldots, \rho_{D}\right)$ as the corresponding vector of computed densities. We refer to the pair $(\mathbf{T}, \boldsymbol{\rho})$ as a dataset, for which we assume the model

$$
\rho=\rho_{\mathcal{H}}(\phi, \mathbf{T})+\boldsymbol{\eta},
$$

where $\phi$ is to be determined, and $\boldsymbol{\eta}$ is a multivariate Gaussian random noise vector. Inspection of residuals-i.e., the difference between the best-fit hyperbola model and the simulated density data-reveals neither bias nor visible correlation; therefore we assume that $\boldsymbol{\eta}$ has zero mean and independent entries. We also observe that the simulation noise is

\footnotetext{
${ }^{5}$ Note, hyperbola-based regression has previously been proposed as a method of interpolating bilinear response functions in Refs. [27, 28]. To the best of our knowledge, such methods have yet to be extended to analysis of quantities in materials science.
} 
larger at higher temperatures. Thus we model the covariance by $\operatorname{Cov}(\boldsymbol{\eta})=\operatorname{diag}\left(\sigma_{1}^{2}, \ldots, \sigma_{\mathrm{D}}^{2}\right)$, where $\sigma_{j} \sim T_{j}^{p}$ is an increasing sequence and $p>0$ is a constant to be determined. We return to this temperature scaling in Sec. 5.1. ${ }^{6}$

Given a dataset $(\mathbf{T}, \boldsymbol{\rho})$, we estimate the parameter $\phi$ by solving the non-linear least-squares problem

$$
\phi_{\mathrm{LS}}=\operatorname{argmin}_{\phi} \sum_{j=1}^{D}\left(\frac{\rho_{\mathcal{H}}\left(\phi, T_{j}\right)-\rho_{j}}{\sigma_{j}}\right)^{2},
$$

which is equivalent to the maximum likelihood estimate of $\phi_{35}^{350}$ under the noise model (2). We have found that given suitable ${ }_{352}$ bounds on the range of parameters, Eq. (3) may be solved using standard algorithms in Matlab (lsqcurvefit). Henceforth we $_{354}$ assume that $\phi$ has been determined and omit the dependence of ${ }_{355}$ $\rho_{\mathcal{H}}$ on this set of parameters.

For a given hyperbola we can define $T_{\mathrm{g}}$ as either (i) the in- ${ }^{356}$ tersection of two tangents to the hyperbola drawn at a low and ${ }_{358}$ high temperature (here $100 \mathrm{~K}$ and $800 \mathrm{~K}$ ), or (ii) as $T_{0}$, the ${ }_{359}$ temperature coordinate of the hyperbola center. Provided that the dataset exhibits asymptotic behavior, these two should be nearly identical. For this reason, we choose neither, but rather 360 compare both estimates to verify that simulated results exhibit the asymptotic behavior expected from theory. We expand upon 361 such consistency tests in the following section.

\subsection{Screening criterion}

The time scales of MD simulations are such that the incred- ${ }^{365}$ ibly rapid cooling rate in silico can distort density-temperature ${ }^{366}$ curves near the glass transition. In some cases, this distortion is ${ }^{367}$ so great that the bilinear characteristics assumed by definition ${ }^{368}$ are not observed. Common practice discards such simulations ${ }^{369}$ on a case-by-case basis. In this section, we propose an algorith- ${ }^{370}$ mic criterion to systematically verify that simulated data $(\mathbf{T}, \boldsymbol{\rho})^{371}$ are consistent with the definition of $T_{\mathrm{g}}$ based on a density- ${ }^{372}$ temperature relationship. Datasets that do not satisfy this cri- $^{373}$ teria can be held-out for closer examination or discarded. Such ${ }^{374}$ situations could occur, for example, if a simulation is unable to ${ }^{375}$ anneal a structure to a physically meaningful energy minimum. ${ }^{376}$

We begin by recalling that the hyperbola described by Eq. (1 $)^{377}$ undergoes a smooth, monotonic transition from an initial slope ${ }^{378}$ of $-a$ to a final slope of $-a-b$. This motivates monitoring the percent convergence of the slope at temperature $T$ relative to its ${ }^{379}$ limiting value as $T \rightarrow \infty$. Specifically, let

$$
\mathcal{P}(T)=\frac{-\rho_{\mathcal{H}^{\prime}}(T)+a}{b}=\frac{1}{2}+\frac{\left(T-T_{0}\right)}{2 \sqrt{\left(T-T_{0}\right)^{2}+4 \mathrm{e}^{c}}},
$$

where $\rho \mathcal{H}_{\mathcal{H}}^{\prime}(T)=\frac{\mathrm{d} \rho_{\mathcal{H}}}{\mathrm{d} T}$, and the factor of $b$ in the denomina- ${ }^{384}$ tor is the difference of the asymptote slopes. By symmetry, it is straightforward to show that the convergence relative to $_{387}$ the lower asymptote is $1-\mathcal{P}(T)$; e.g., $95 \%$ converged to one ${ }_{388}$ asymptote is $5 \%$ converged to the other.

${ }^{6}$ We have found that the precise value of $p$ has little effect on the fits, provided $1 / 2 \lesssim p \lesssim 2$.
Next, we introduce a user-defined parameter $\hat{\mathcal{P}}$ expressing a minimum amount of convergence necessary for a dataset to be considered acceptable. Replacing $\mathcal{P}(T)$ by $\hat{\mathcal{P}}$, we invert Eq. (4) to find a temperature interval over which $\rho(T)$ is transitioning. Specifically, computing

$$
\Delta T=\frac{\mathrm{e}^{c / 2}(2 \hat{\mathcal{P}}-1)}{\sqrt{\hat{\mathcal{P}}(1-\hat{\mathcal{P}})}},
$$

we define $T_{-}=T_{0}-\Delta T$ and $T_{+}=T_{0}+\Delta T$. The interval $T_{-}<T<T_{+}$then bounds those temperatures for which the hyperbola slope is less than $\hat{\mathcal{P}}$-percent converged to its asymptotes. Provided that the interval of simulation temperatures extends beyond this transition interval on both sides, the simulation contains data in the asymptotic regimes. Otherwise, one or more of the asymptotic regimes is not exhibited, and we hold out the dataset for further inspection or rejection. Figure 3 illustrates the use of this rejection criterion when $\hat{\mathcal{P}}=90 \%$ (indicated by the "transition region" in the figure).

\section{Pooling and the thermodynamic limit}

Experimentally, bulk mechanical properties are typically measured using samples whose masses are a few grams or more, corresponding to numbers of atoms on the order of Avogadro's number, $N=O\left(10^{23}\right)$. Such sizes are well out of reach of atomistic MD, which is generally restricted to systems having $N \lesssim O\left(10^{6}\right)$. Given this disparity of scales, it is natural to ask whether $T_{\mathrm{g}}$ estimates obtained by atomistic simulation have converged to their bulk counterparts.

From the outset, this question appears intractable, as we cannot realize bulk simulations. However, statistical mechanics provides information about the dispersion of intensive quantities (such as the density) in the thermodynamic limit $N \rightarrow \infty$. From this, one can deduce corresponding properties of $T_{\mathrm{g}}$. As we show, this yields a set of criteria that should hold for any converging estimate of $T_{\mathrm{g}}$. In this section, we (i) describe the derivation of these criteria and (ii) show how a pooling analysis can demonstrate when these convergence criteria are not satisfied.

\subsection{Underlying ideas: the role of linearization}

In statistical mechanics, asymptotic expansions for intensive quantities (such as density) may be derived as $N \rightarrow \infty$. In particular, it is well known that: (i) the expected value $\bar{\rho}(T)$ is independent of $N$; and (ii) fluctuations about $\bar{\rho}(T)$ between independent realizations of a system are normally distributed with a variance that is proportional to $1 / N$. The former is usually taken as a definition of "intensive," while the latter can formally be viewed as a consequence of the Central Limit Theorem. See, for example, Ref. [30].

To frame this idea in the context of simulations, we recast Eq. (2) by assuming

$$
\boldsymbol{\rho}(N)=\overline{\boldsymbol{\rho}}+\boldsymbol{\eta}(N),
$$



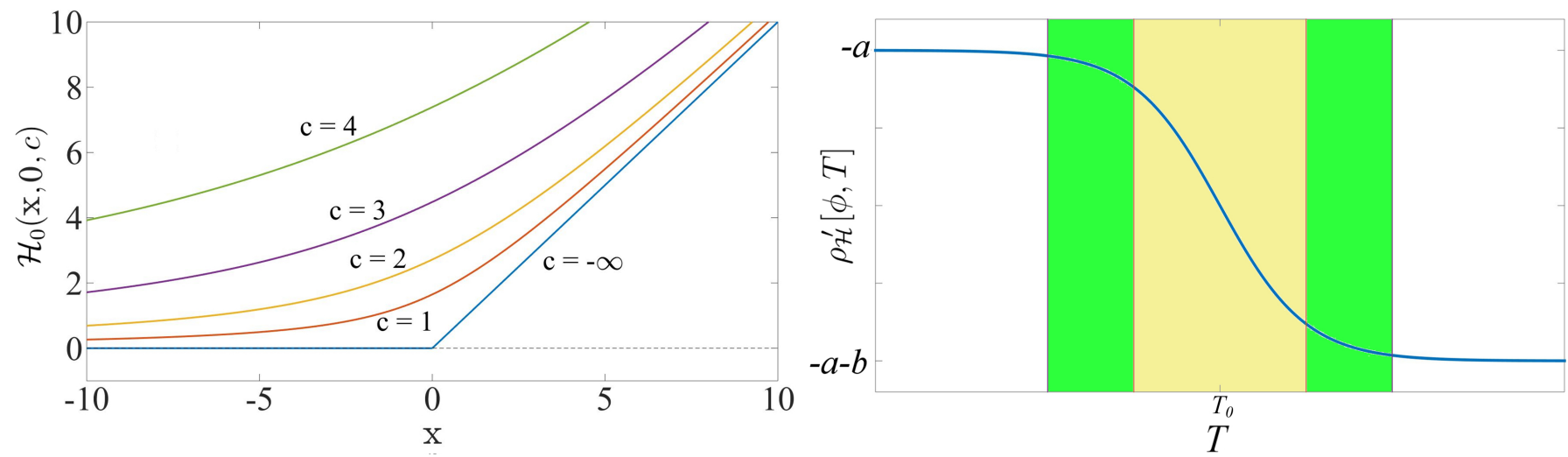

Figure 2: Left plot: the hyperbola $\mathcal{H}_{0}(x, 0, c)$ for different values of $c$. As $c \rightarrow-\infty$, the hyperbola converges to a piecewise linear function. Right plot: an example the derivative $\rho_{\mathcal{H}}^{\prime}(T)$ as a function of $T$. The slope of the hyperbola changes monotonically from $-a$ to $-a-b$. The yellow and green shaded areas correspond to transition regions beyond which the hyperbola slope has converged to more than $90 \%$ and $98 \%$ of its asymptotic values, respectively.
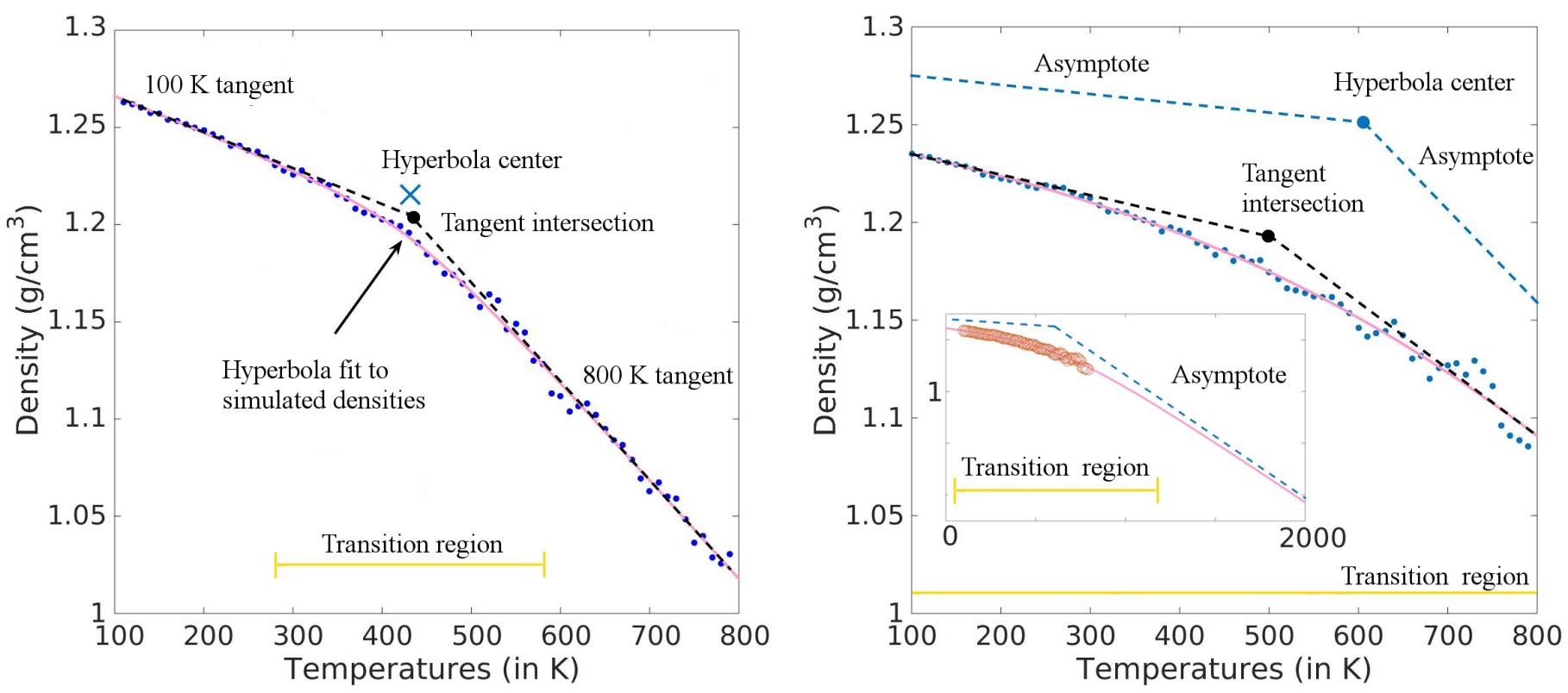

Figure 3: Hyperbola fits to MD simulation data. The transition region indicates all of the data for which the hyperbola is less than $90 \%$ converged to either asymptote. Left plot: hyperbola (pink) fit to the 3800 atom 33BF data shown in Fig. 1. Here $T_{\mathrm{g}}$ is given by the $T$ coordinate of either the hyperbola center or the intersection of the tangents computed at $100 \mathrm{~K}$ and $800 \mathrm{~K}$. For this set of data, the tangents and asymptotes are virtually indistinguishable. Right plot: Hyperbola fit to data from a 4400 atom 3344MY simulation; note that fluctuations are much larger than for the 33BF data. The dashed black lines are the low and high temperature tangents. The hyperbola center lies far above the data. The inset shows the same data on an expanded scale, revealing an incomplete transition to the asympotic regime. Compare with the left plot for 33BF, where the hyperbola asymptotes and tangents essentially overlap. 
where $\bar{\rho}$ is independent of $N, \boldsymbol{\eta}(N)$ is a zero-mean, Gaussian ${ }_{420}$ random variable, and $\operatorname{Var}[\boldsymbol{\eta}(N)] \sim 1 / N$ as $N \rightarrow \infty$. Equa-421 tion (6) implies that the density-temperature relationship becomes deterministic as the system size goes to infinity. Given that we cannot simulate such a system, the quantities $\bar{\rho}$ and $\boldsymbol{\eta}$ are unknown and must be estimated. (Here, as above, bold symbols are used to refer to quantities evaluated on a fixed temperature grid.)

Ideally we could show that $T_{\mathrm{g}}$ estimates obtained from atomistic-scale simulations are representative of an estimate derived from Eq. (6); this would lend confidence to the correspondence between simulations and bulk systems. Since $\bar{\rho}$ and $\boldsymbol{\eta}$ are unknown, it is necessary to pursue a weaker and indirect confirmation by comparing the $N$-dependence of $T_{\mathrm{g}}$ with asymptotic predictions. Denote by $\mathcal{H}[\bar{\rho}]$ the non-linear regression analysis that estimates $T_{\mathrm{g}}$ from density data. Note that the large- $N$ [i.e., small dispersion in $\rho(N)$ ] expansion for $\mathcal{H}$ may be approx- ${ }^{422}$ imated by linearization

$$
\begin{aligned}
T_{\mathrm{g}}=\mathcal{H}[\boldsymbol{\rho}] & =\mathcal{H}[\overline{\boldsymbol{\rho}}]+\boldsymbol{\eta} \cdot \nabla_{\boldsymbol{\rho}} \mathcal{H}+\mathcal{O}\left(\|\boldsymbol{\eta}\|^{2}\right) \\
& \approx \bar{T}_{\mathrm{g}}+\boldsymbol{\eta} \cdot \nabla_{\boldsymbol{\rho}} \mathcal{H}
\end{aligned}
$$

where $\nabla_{\rho}$ is the gradient taken with respect to densities, and we identify $\mathcal{H}[\bar{\rho}]=\bar{T}_{\mathrm{g}}$. Subject to the validity of the linear approximation, Eq. (7), one finds

$$
\begin{aligned}
\mathbf{E}\left[T_{\mathrm{g}}\right] & \approx \mathbf{E}\left[\bar{T}_{\mathrm{g}}+\boldsymbol{\eta} \cdot \nabla_{\rho} \mathcal{H}\right]=\bar{T}_{\mathrm{g}} \\
\operatorname{Var}\left[T_{\mathrm{g}}\right] & \approx \operatorname{Var}\left[\boldsymbol{\eta} \cdot \nabla_{\rho} \mathcal{H}\right] \sim 1 / N .
\end{aligned}
$$

Equations (8) and (9) provide a set of criteria that should hold ${ }_{426}$ for any $T_{\mathrm{g}}$ estimation method: the expected value should be ${ }_{427}$ independent of $N$, and the variance should decay as $1 / N$.

In view of Eq. (7), there are at least two ways in which an estimate can fail to satisfy Eqs. (8) and (9): (i) $\bar{\rho}=\bar{\rho}(N)$ depends on $N$; or (ii) the variance of $\boldsymbol{\eta}$ is so large that $O\left(\|\eta\|^{2}\right)$ terms are not negligible. Tests for the former require that we simulate multiple system sizes. We explore this approach in Sec. 4.2. Condition (ii) is easier to check, although its effects are less obvious.

To illustrate this last point, we take the expectation of Eq. (7), and retain the $O\left(\|\boldsymbol{\eta}\|^{2}\right)$ terms. This yields

$$
\mathbf{E}\left[T_{\mathrm{g}}\right]=\bar{T}_{\mathrm{g}}+\mathbf{E}\left[O\left(\|\boldsymbol{\eta}(N)\|^{2}\right)\right] .
$$

From this we see that large noise will result in an $N$-dependent bias to our $T_{\mathrm{g}}$ estimates obtained by our non-linear regression. To the extent that this bias is significant, one anticipates that simulating different system sizes will result in different ex-428 pected values of $T_{\mathrm{g}}$. Analogously, deviations from the $1 / N^{429}$ scaling of the variance would also be indicative of a lack of ${ }^{430}$ convergence.

\subsection{Data pooling}

Motivated by the considerations of the previous section, $\mathrm{we}^{434}$ pursue methods that assess whether corrections to Eqs. (8) and (9) are observable. In particular, we note that applying $\mathcal{H}$ to a sequence of datasets with the same mean densities but decreasing variances should reveal the order of $\boldsymbol{\eta}$ dependence in
Eq. (7). We therefore look for ways to combine our datasets into effective data pools having the above properties.

First, assume that we have $M$ total datasets $\boldsymbol{\rho}_{i}, 1 \leq i \leq M$, and denote $T_{\mathrm{g}, i}=\mathcal{H}\left[\boldsymbol{\rho}_{i}\right]$ as the value of $T_{\mathrm{g}}$ associated with the $i$-th dataset. ${ }^{7}$ We define a data pool via the sample average

$$
\rho_{\alpha}:=\frac{1}{|\alpha|} \sum_{i \in \alpha} \rho_{i}
$$

where $\alpha$ is a pool denoting a set of indices $\alpha:=\left\{i_{1}, i_{2}, \ldots, i_{|\alpha|}\right\}$, and $|\alpha|$ is the cardinality of $\alpha$ (i.e., the number of elements $\alpha$ ). Importantly, we distinguish the following averages

$$
T_{\mathrm{g}}(\alpha):=\mathcal{H}\left[\rho_{\alpha}\right] \neq \frac{1}{|\alpha|} \sum_{i \in \alpha} \mathcal{H}\left[\rho_{i}\right]=\frac{1}{|\alpha|} \sum_{i \in \alpha} T_{\mathrm{g}, i},
$$

since $\mathcal{H}$ is not linear.

We view $\boldsymbol{\rho}_{\alpha}$ and $T_{g}(\alpha)$ as estimates coming from a system that is $|\alpha|$ times larger than any of those associated with the base quantities $\rho_{i}$ (or $T_{\mathrm{g}, i}$ ). Taking this to be true for the time being, we observe that, by definition, $\mathbf{E}\left[\rho_{\alpha}\right]=\mathbf{E}\left[\boldsymbol{\rho}_{i}\right]=\overline{\boldsymbol{\rho}}$, independent of $|\alpha|$, and

$$
\operatorname{Var}\left[\rho_{\alpha}\right]=\operatorname{Var}\left[\sum_{i \in \alpha} \frac{\boldsymbol{\rho}_{i}}{|\alpha|}\right]=\frac{\operatorname{Var}\left[\rho_{i^{\prime}}\right]}{|\alpha|},
$$

for any $i^{\prime} \in \alpha$. Note that this is not a consequence of any thermodynamic limiting process, but rather follows from linearity and the assumption that $\rho_{i}$ are independent and identically distributed. Thus, by construction, our data pools $\rho_{\alpha}$ have the properties discussed above.

To make use of this, let $A_{m}=\{\alpha:|\alpha|=m\}$ be a set of $\alpha$ with fixed cardinality. Next, define

$$
\tilde{T}_{\mathrm{g}}(m):=\frac{1}{\left|A_{m}\right|} \sum_{\alpha \in A_{m}} T_{\mathrm{g}}(\alpha) .
$$

Note that $A_{m}$ need not exhaust all combinations of sets, and the elements in $A_{m}$ may share datasets in common (i.e. $\alpha$ and $\alpha^{\prime} \neq \alpha$ may be dependent). Given Eq. (14), we also define the variance

$$
\begin{aligned}
s^{2}(m) & :=\frac{1}{f_{m}\left|A_{m}\right|} \sum_{\alpha \in A_{m}}\left(T_{\mathrm{g}}(\alpha)-\tilde{T}_{\mathrm{g}}(m)\right)^{2} \\
f_{m} & :=1-\sum_{\alpha, \alpha^{\prime} \in A_{m}} \frac{\left|\alpha \cap \alpha^{\prime}\right|}{m\left|A_{m}\right|^{2}},
\end{aligned}
$$

where $\alpha \cap \alpha^{\prime}$ denotes the intersection of $\alpha$ and $\alpha^{\prime}$, i.e. the elements shared by both sets.

It is possible to show that $\tilde{T}(m)$ is constant and $s^{2}(m) \propto 1 / m$ when $\mathcal{H}$ is linear and $A_{m}$ exhausts all combinations. Importantly, the contrapositive therefore also holds: if $\tilde{T}(m)$ is not constant or $s^{2}(m) \not<1 / m$, then $\mathcal{H}$ is not linear; cf. the Appendices. Thus, a simple test for quadratic corrections to Eq. (7)

\footnotetext{
${ }^{7}$ Note, here we consider $T_{\mathrm{g}, i}$ as a point estimate with no uncertainties, since our aim is to assess the validity of Eq. (7). In Sec. 5.2, we reintroduce uncertainties when constructing our final $T_{\mathrm{g}}$ estimate.
} 


\section{Uncertainty estimates and consensus value of $T_{\mathrm{g}}$}

In Eq. (6) of the previous section, we proposed a generic ${ }_{494}$ model for simulated density-temperature data and discussed ${ }_{495}$ how noise $\boldsymbol{\eta}$ can propagate through the hyperbola fits. Here we pursue a refinement of this model, partitioning $\boldsymbol{\eta}$ into two terms with different physical origins. The main idea is to isolate shared contributions from those that are unique to each dataset. In particular, we postulate that Eq. (6) can be further refined into

$$
\boldsymbol{\rho}_{i}(N)=\overline{\boldsymbol{\rho}}+\boldsymbol{\omega}_{i}(N)+\boldsymbol{\beta}_{i}(N),
$$

where the subscript $i$ indexes the dataset, $\omega_{i}$ is a withinsimulation noise, $\boldsymbol{\beta}_{i}$ is a between-simulation noise. The motivation for identifying $\boldsymbol{\eta}_{i}=\omega_{i}+\boldsymbol{\beta}_{i}$ is illustrated in Fig. 6, where we superimpose density-temperature curves from multiple simulations. Two features are apparent: (i) each curve exhibits random fluctuations about a hyperbola-like shape; and (ii) the curves appear to be approximately shifted relative to one another. We model the first of these with $\omega_{i}$ and attribute the associated fluctuations to finite-size and -time effects specific to each simulation. We model the second with $\boldsymbol{\beta}_{i}$ and associate this uncertainty with limited sampling of the crosslinked network and limited exploration of the energy landscape.

Given Eq. (17), we may formally propagate $\omega_{i}+\boldsymbol{\beta}_{i}$ through $\mathcal{H}$ [as in Eq. (7)] to find a mixed effects model

$$
T_{\mathrm{g}, i}=\bar{T}_{\mathrm{g}}+\tau_{\omega, i}+\tau_{\beta, i}
$$

where $\tau_{\omega, i}=\omega_{i} \cdot \nabla_{\rho} \mathcal{H}$ and $\tau_{\beta, i}=\beta_{i} \cdot \nabla_{\rho} \mathcal{H}$; note that these identities rely on the approximate linearity of $\mathcal{H}$. For simplicity, we assume that $\tau_{\omega, i}=\mathcal{N}\left(0, \varsigma_{i}^{2}\right)$ and $\tau_{\beta, i}=\mathcal{N}\left(0, y^{2}\right)$, where $\mathcal{N}(0, X)$ is a zero-mean Gaussian random variable with variance $X$ and $\varsigma_{i}^{2}$ depends on the $i$ th dataset.

In the following two sections, we discuss methods for estimating the dispersions $\tau_{\omega, i}$ and $\tau_{\beta, i}$, and combining them to construct a final estimate of $T_{\mathrm{g}}$. We first estimate $\tau_{\omega, i}$, which must be obtained individually for each dataset (Sec. 5.1). Given the within-uncertainties, we then proceed to an analysis of $\tau_{\beta, i}$ (Sec. 5.2).

\subsection{Noise propagation and within-simulation uncertainty}

Any estimate of $T_{\mathrm{g}}$, computational or experimental, is incomplete without an associated uncertainty [31]. In our case, the $\rho(T)$ curves obtained from MD simulations are noisy due to fluctuations in the underlying dynamics and finite-time, finitesize effects. In this section, we propagate this noise into associated uncertainties in $T_{g}$ via a sampling technique akin to parametric bootstrap analysis in statistics [32].

In more detail, let $\left(\mathbf{T}, \boldsymbol{\rho}_{i}\right)$ denote a fixed dataset, with $\boldsymbol{\rho}_{i}=$ $\left(\rho_{i, 1}, \ldots, \rho_{i, D}\right)$ and $j(1 \leq j \leq D)$ indexing data-points within the set. In light of Eqs. (17) and (18), the between-noise $\boldsymbol{\beta}_{i}$ appears as a fixed term that can be temporarily absorbed into $\bar{\rho}$. Thus we consider the reduced models

$$
\begin{aligned}
\boldsymbol{\rho}_{i} & =\hat{\boldsymbol{\rho}}_{i}(T)+\omega_{i}(T), \\
T_{\mathrm{g}, i} & =\hat{T}_{\mathrm{g}, i}+\tau_{\omega, i},
\end{aligned}
$$

where the notation $\hat{\boldsymbol{\rho}}_{i}$ and $\hat{T}_{\mathrm{g}, i}$ indicates that averages between datasets differ by virtue of the $\boldsymbol{\beta}_{i}$. We seek $\boldsymbol{\zeta}_{i}^{2}=\operatorname{Var}\left[\tau_{\omega, i}\right]$ as a quantification of the within-simulation uncertainty in $T_{\mathrm{g}}$.

There are many ways to estimate $\operatorname{Var}\left[\tau_{\omega, i}\right]$. Here we use a synthetic data approach based on repeated samples of the noise $\omega_{i}$. For this we postulate a multi-variate Gaussian model for $\omega_{i}$ and infer the underlying parameters from the given dataset. As reflected in Eq. (2), we interpret the residuals $\boldsymbol{\rho}_{i}-\rho_{\mathcal{H}}\left(\phi_{\mathrm{LS}}, \mathbf{T}\right)$ as a sample of $\omega_{i}$. Taking a fixed polymer system of modest size, we compute ten independent annealing runs and superimpose the residuals as a function of temperature. A few observations can be made. First, there is no discernible bias in the residuals. This is significant as the hyperbolic fit, being a global model for $\rho(T)$ with relatively few parameters, imposes nontrivial structure on the computational data. Departure from this structure would be observable as bias. As we visually observe no bias we conclude that the hyperbola model is suitable and, furthermore, $\omega_{i}$ may be assumed to have zero mean. Second, as there are neither clear indications nor theoretical motivations for correlations between densities at different temperatures, we assume zero correlation, i.e., $\operatorname{Cov}\left(\omega_{i}\right)=\operatorname{diag}\left(\sigma_{i, j}^{2}\right)$; note that diagonalization occurs over $j$, which indexes temperature [cf. text under Eq. (2)]. Finally, the residuals show increased noise at higher temperatures. Intuitively this is reasonable from thermodynamic considerations, and furthermore motivates a powerlaw dependence

$$
\sigma_{i, j}=T_{j}^{p} \sigma_{*, i} .
$$



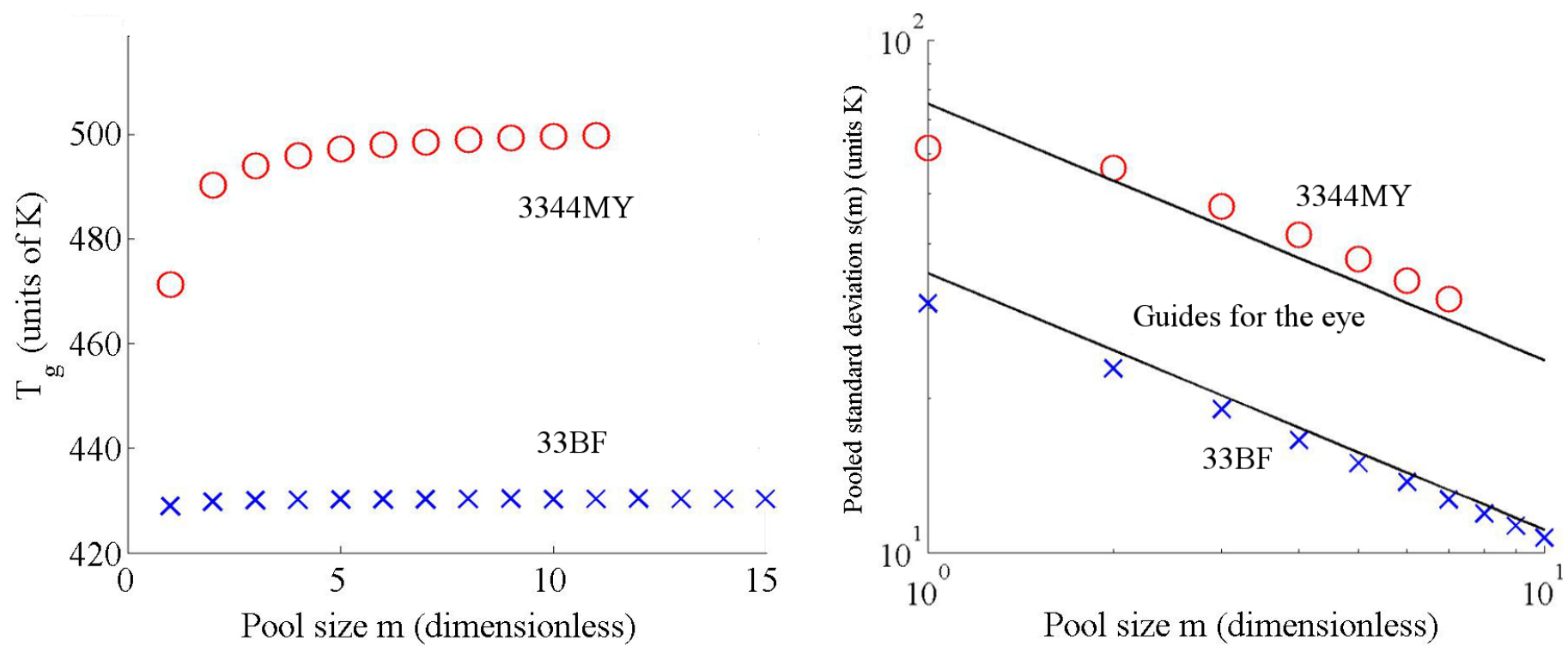

Figure 4: Pooled averages and standard deviations of $T_{\mathrm{g}}$ for 3800 atom 33BF and 4400 atom $3344 \mathrm{MY}$ simulations. All estimates were computed using the hyperbola center method, and datasets were only pooled if their asymptotic regimes satisfied the $\hat{P}=90 \%$ screening criterion. The data available after screening amounted to 40 sets for $33 \mathrm{BF}$ cured at $725 \mathrm{~K}, 12$ sets for $3344 \mathrm{MY}$ cured at $350 \mathrm{~K}$, and 8 sets for $3344 \mathrm{MY}$ cured at $725 \mathrm{~K}$. Left plot: Comparison of pooled $T_{\mathrm{g}}$ values from simulations of 33BF cured at $725 \mathrm{~K}$ and $3344 \mathrm{MY}$ cured at $350 \mathrm{~K}$. The $33 \mathrm{BF}$ estimates are roughly independent of pool size, whereas the $3344 \mathrm{MY}$ estimates are not. Right plot: Comparison of pooled standard deviations from simulations of 33BF cured at $725 \mathrm{~K}$ and $3344 \mathrm{MY}$ cured at $725 \mathrm{~K}$. The solid black lines have a slope of $-1 / 2$ and are guides for the eye. The 33BF estimates follow the power law closely for all pool sizes, whereas 3344MY estimates do not.
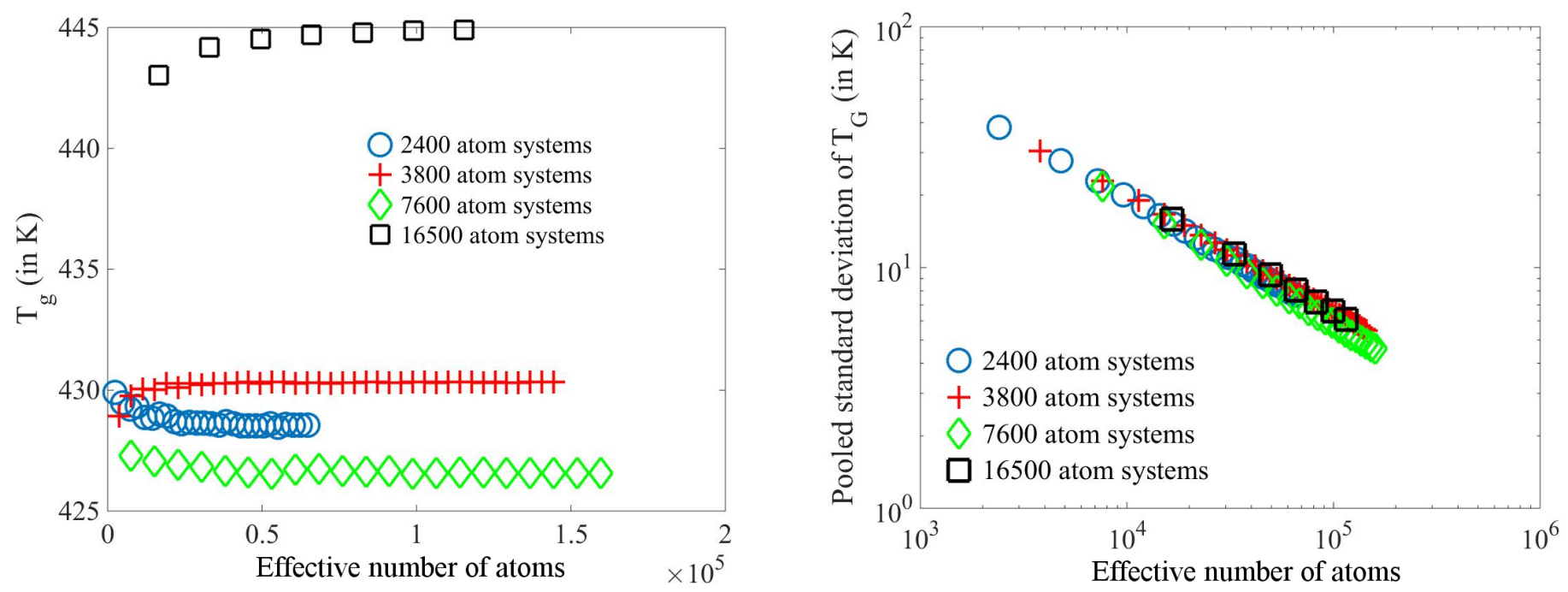

Figure 5: Comparison of pooled results with original datasets from 33BF simulations of different sizes. Datasets from different sized simulations are never added to the same pool so that we may more clearly assess the convergence of $\bar{\rho}(N)$ as a function of simulation size. Left plot: Pooled $T_{\mathrm{g}}$ estimates as a function of effective number of atoms. All four system sizes yield well converged estimates that are within about $15 \mathrm{~K}$ of one another. Right plot: Pooled standard deviations as a function of effective number of atoms. The data collapse suggests that pool size is an appropriate proxy for system size. Given the uncertainties in the right plot, the corresponding error bars of the left plot would overlap. 

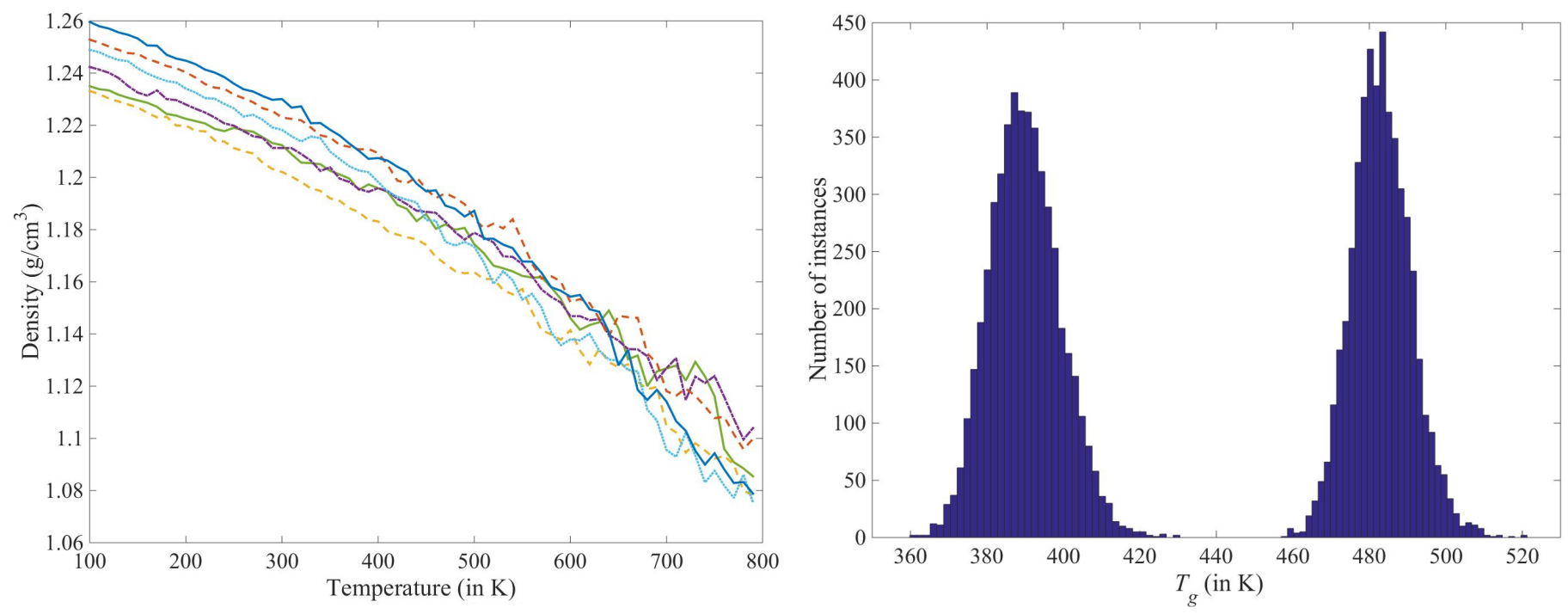

Figure 6: Comparison of simulated results from different realizations of the same system. Left plot: six different density-temperature curves generated from independent realizations of 4400-atom 3344MY simulations. Right plot: histograms of $T_{\mathrm{g}}$ values from the hyperbola method applied to synthetic data (cf. Sec. 5.1) generated from two independent 3800-atom 33BF datasets (i.e., different simulations of the same chemistry). The lack of overlap between the distributions suggests that the noise propagation model does not capture all of the uncertainty in $T_{\mathrm{g}}$.

Empirically, we find that setting $p=5 / 4$ yields scaled residuals that have approximately a uniform variance; see Fig. 7, which is representative of our computational datasets. For $p$ fixed we estimate the overall scale of the variance by

$$
\sigma_{*, i}^{2}:=\frac{1}{D} \sum_{j=1}^{D} \frac{\left[\rho\left(\phi_{\mathrm{LS}}, T_{j}\right)-\rho_{i, j}\right]^{2}}{T_{j}^{2 p}}
$$

With $\omega_{i}$ fully specified, we generate the synthetic datasets $\hat{\boldsymbol{\rho}}_{i, k}=\rho\left(\phi_{\mathrm{LS}}, \mathbf{T}\right)+\boldsymbol{\omega}_{i, k}$ using a pseudorandom number generator. Evaluation of $\mathcal{H}\left[\hat{\boldsymbol{\rho}}_{i, k}, \mathbf{T}\right]$ results in an empirical distribution for $\omega_{i}$, from which we compute $\hat{T}_{\mathrm{g}, i}$ and its variance.

The left subplot of Fig. 8 shows the the results of sampling 20000 synthetic datasets $\hat{\boldsymbol{\rho}}_{i, k}$ for the simulated 33BF results of Fig. 3. Both estimation methods based on the hyperbola regression - the hyperbola center, and the intersection of the two tangents-yield essentially overlapping distributions, consistent with the asymptotic-regime convergence test.

The right subplot of Fig. 8 shows the results of sampling 20000 synthetic sets for the 3344MY simulation that generated Fig. 3. Even though we would normally reject this simulation for failing to sample asymptotic regimes, it is instructive to consider how the underlying noise propagates through the fit. Clearly, the distributions of $T_{\mathrm{g}}$ associated with the two hyperbola methods differ significantly, reflecting the fact that the densities only sample the transition region. This further supports the conclusion that we cannot confidently extract a $T_{\mathrm{g}}$ value from this simulation.

The right subplot of Fig. 6 shows two histograms computed from independent realizations of 3800-atom 33BF datasets. The lack of overlap in the distributions is yet another indicator that the between-simulation noise discussed in Eq. (17) has a significant effect on the uncertainty in simulated $T_{\mathrm{g}}$ estimates.

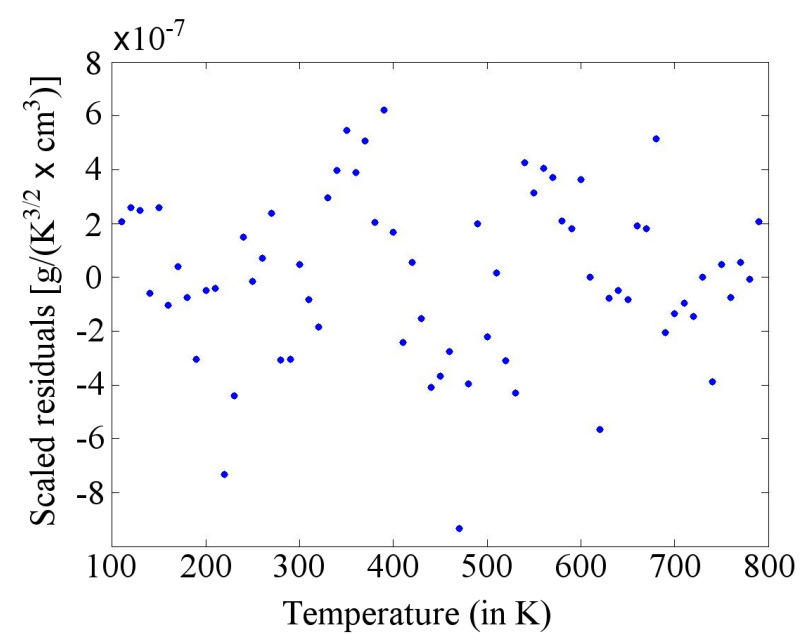

Figure 7: Residuals $\rho(\hat{\phi}, \mathbf{T})-\boldsymbol{\rho}$ after rescaling by $\mathbf{T}^{5 / 4}$. 

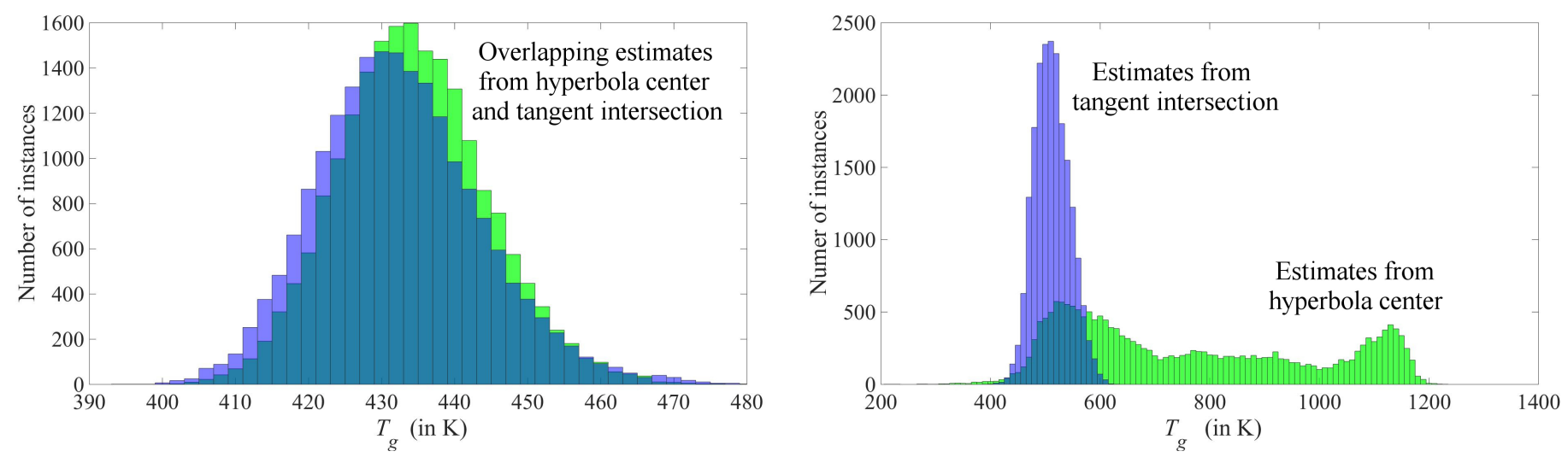

Figure 8: Histograms of $T_{\mathrm{g}}$ calculated from the hyperbola tangent intersection (purple) and hyperbola center (green) applied to synthetic data. Each histogram was generated from 20000 realizations of synthetic datasets according to Eq. (21). Left plot: Histogram based on the 3800 atom 33BF simulation associated with Figs. 1 and 3. The calculations based on both hyperbola methods essentially overlap, consistent with our conclusion that the underlying data samples asymptotic regimes. Right plot: Histogram based on the 4400 atom 3344MY simulation associated with the right subplot of Fig. 3. Disagreement between the two hyperbola methods reinforces the notion that the underlying dataset should be examined more closely and possibly rejected on the grounds that it does not sample asymptotic behavior.

\subsection{Between uncertainty and weighted-mean averages}

530

In the previous section, we presented a noise-propagation ${ }^{531}$ method for estimating the within-simulation uncertainty $\tau_{\omega, i}$. It ${ }^{532}$ remains to determine the between uncertainty $\tau_{\beta, i}$, and to com-533 pile all simulated results for $T_{\mathrm{g}}$ into a single estimate with its ${ }^{534}$ associated (combined) uncertainty.

Denote by $\hat{\mathbf{T}}_{\mathrm{g}}=\left(\hat{T}_{\mathrm{g}, 1}, \ldots, \hat{T}_{\mathrm{g}, M}\right)$ the collection of $M^{536}$ simulation-based estimates of $T_{\mathrm{g}}$. Given our statistical model, ${ }^{537}$ the joint probability density function for $\hat{\mathbf{T}}_{\mathrm{g}}$ is given by

$$
f\left(\hat{\mathbf{T}}_{\mathrm{g}} \mid \bar{T}_{\mathrm{g}}, y\right)=\frac{\exp \left(-\frac{1}{2} \sum_{i} \frac{\left(\hat{T}_{\mathrm{g}, i}-\bar{T}_{\mathrm{g}}\right)^{2}}{s_{i}^{2}+y^{2}}\right)}{(2 \pi)^{M / 2} \sqrt{\prod_{i}\left(s_{i}^{2}+y^{2}\right)}}
$$

Considering the above as a function of $\left(\bar{T}_{\mathrm{g}}, y\right)$ and maximizing the log-likelihood [i.e. the logarithm of Eq. (23)] results in the equations

$$
\begin{array}{r}
\sum_{i=1}^{M} \frac{\hat{T}_{\mathrm{g}, i}-\bar{T}_{\mathrm{g}}}{\varsigma_{i}^{2}+y^{2}}=0 \\
\sum_{i=1}^{M} \frac{1}{\varsigma_{i}^{2}+y^{2}}-\sum_{i=1}^{M} \frac{\left(\hat{T}_{\mathrm{g}, i}-\bar{T}_{\mathrm{g}}\right)^{2}}{\left(\varsigma_{i}^{2}+y^{2}\right)^{2}}=0
\end{array}
$$

for a critical point. Solution to the first motivates estimating ${ }_{541}^{540}$ $T_{\mathrm{g}}$ by a weighted average

$$
\mathcal{T}=\left[\sum_{i=1}^{M} \frac{1}{y^{2}+\varsigma_{i}^{2}}\right]^{-1} \sum_{i=1}^{M} \frac{\hat{T}_{\mathrm{g}, i}}{y^{2}+\varsigma_{i}^{2}} .
$$

Setting $y=0$ results in the usual minimum variance estimator ${ }^{546}$ of $T_{\mathrm{g}}$ as the mean of independent, heteroscedastic random vari- ${ }^{547}$ ables. ${ }^{8}$ However, as is well-known, this estimation suffers from ${ }_{549}^{548}$

\footnotetext{
${ }^{8}$ Heteroskedastic means that each random variable potentially has a different ${ }^{551}$ variance.
}

over-emphasizing experiments (or computations in our case), whose uncertainty $\varsigma_{i}^{2}$ is underestimated. The central benefits of Eq. (26) are that it accommodates additional, unaccounted for uncertainties, referred to colorfully as "dark-uncertainties." In doing so, Eq. (26) also avoids upweighting overconfident results. Note that when $\varsigma_{i}^{2} \ll y^{2}$ for all $i$, Eq. (26) returns the sample average; i.e., the estimator views each realization $T_{i}$ as an equally meaningful draw from a statistical ensemble. For more details on Eq. (26) we refer to Ref. [33] and its many references.

It remains to determine the quantity $y$. Several approaches have been recommended; see Ref. [33] for a review. In our case, we complete the maximum likelihood analysis by substituting the weighted mean Eq. (26) into Eq. (25), and solving the nonlinear system for $y$. Having determined the model parameters $\mathcal{T}, y$, and $\varsigma_{i}$, Ref. [33] provides a consistent estimator for the variance of $\mathcal{T}$ having the form

$$
\delta^{2}=\left[\sum_{i=1}^{M} \frac{1}{y^{2}+\varsigma_{i}^{2}}\right]^{-2} \sum_{i=1}^{M} \frac{\left(\hat{T}_{\mathrm{g}, i}-\mathcal{T}\right)^{2}}{\left(y^{2}+\varsigma_{i}^{2}\right)^{2}} .
$$

Notably, $\delta$ has the property that $\delta \rightarrow 0$ as the number of simulations $M \rightarrow \infty$, provided the $\varsigma_{i}$ are well behaved. We use $\delta$ to indicate the confidence with which we have determined the true $T_{\mathrm{g}}$ value predicted by arbitrarily large MD simulations of a given chemistry. That is, we approximate the $99 \%$ confidence interval for $T_{\mathrm{g}}$ to be $\mathcal{T} \pm 3 \delta$.

In Fig. 9, we show $T_{g}$ estimates for individual 33BF datasets according to Eq. (26). Note that many datasets yield predictions whose error bars fail to overlap. The between-simulation uncertainty is shown on the right side of the plot and illustrates that $y$ accounts for this lack of consistency between individual simulations. We also plot the $99 \%$ confidence interval $\mathcal{T} \pm 3 \delta$, which is small by virtue of the fact that $M$ is relatively large. 


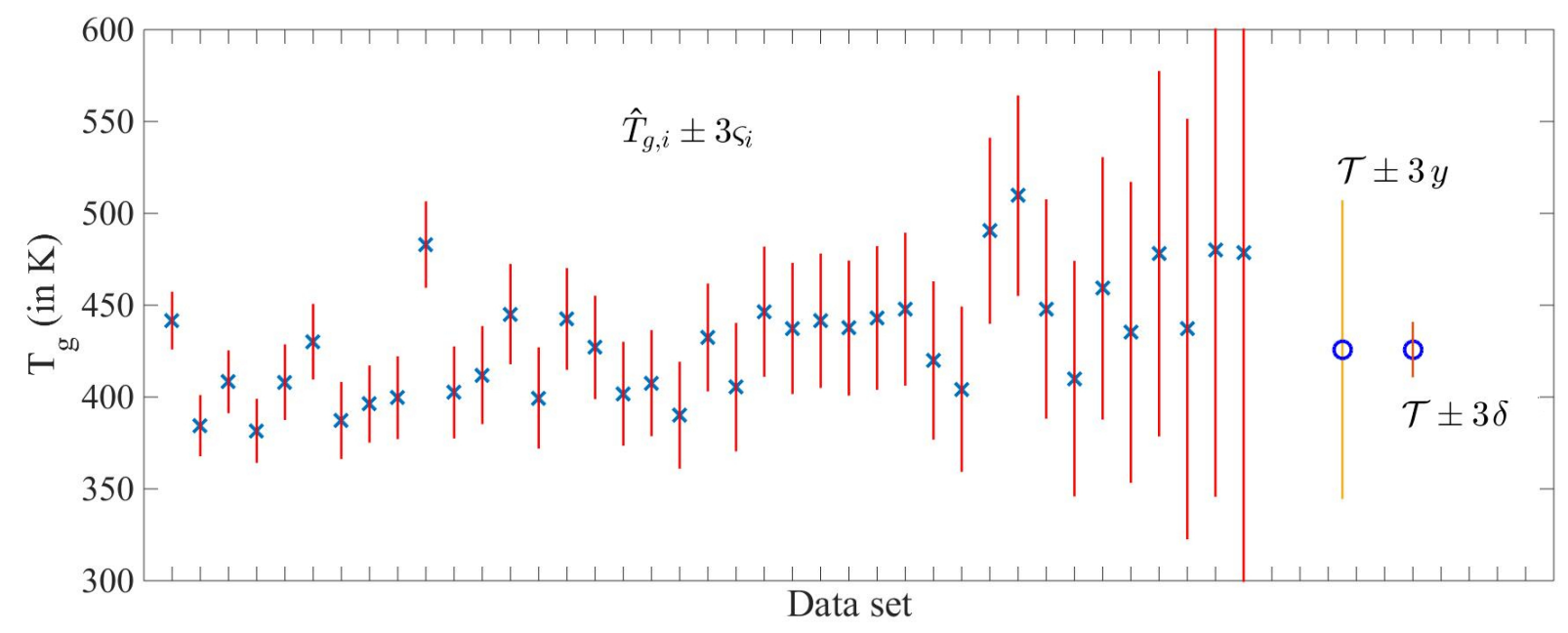

Figure 9: 33BF $T_{\mathrm{g}}$ estimates from individual datasets and according to Eq. (26). Blue $\times$ indicate hyperbola analyses applied to individual datasets; red error bars correspond to $3 \varsigma_{i}$ values of the within-simulation uncertainty. The blue o correspond to $T_{\mathrm{g}}$ estimates $\mathcal{T}$ via Eq. (26). The corresponding gold error bar (second from right) corresponds to $\pm 3 y$, illustrating that the between-simulation uncertainty $y$ accounts for finite-size and finite-time effects between individual realizations. The short orange error bar (far right) corresponds to $\pm 3 \delta$. It is small by virtue of the fact that we reduce uncertainty by combining results from many simulations.

To the best of our knowledge, there are few works that explic-589 itly discuss verification of simulated predictions for $T_{\mathrm{g}}$. Refer-590 ences [20-22] are notable exceptions. In Refs. [20, 21] the au-591 thors estimated uncertainties in bilinear-fit estimates of $T_{\mathrm{g}}$ us-592 ing a synthetic data approach similar to the one we propose. 593 However, their analogue to our $\boldsymbol{\eta}$ was constructed from varia-594 tions in density between simulations, and the subsequent analy-595 sis did not differentiate types of uncertainty as we have. Refer-596 ence [22] also identified asymptotic regimes by examining the597 behavior of numerical approximations of derivatives in density-598 temperature data. Our analysis is distinct insofar as it provides 599 an algorithmic screening criterion based on analytical proper-600 ties of the hyperbola regression model.

We also note that the pooling analysis of Sec. 4.2 is similar6o2 in spirit to the Jackknife estimator proposed, e.g., in Ref. [34].603 The goal of that work was to provide estimators that reduce bias604 in non-linear estimation problems.

\subsection{Limitations}

All of our analyses-hyperbola regression and transition anal- $-^{608}$ ysis, noise propagation, and pooling analysis-can indicate ${ }^{609}$ when we must consider larger systems, but not that a given system is large enough. The most heroic simulations today con- ${ }^{610}$ sider billions of atoms, far shy of the numbers of atoms in a611 physical bulk sample [35]. Unfortunately, direct atomistic sim-612 ulation of bulk scales is currently out of reach and will remain ${ }_{613}$ so for some time to come. From a modeling perspective, how-614 ever, the problem is less bleak. Recent significant advances 615 in formulating coarse-grained models of discrete polymer sys-616 tems (e.g. as in Ref. [36]) could potentially lead to a better un-617 derstanding of what system size is sufficient to model bulk be-618 havior. Nonetheless, such studies lie beyond the scope of our 619 work, and for now we caution practitioners that other considerations might enforce stricter lower bounds on system size than the methods we propose.

Our analysis omits quantification of parametric uncertainty built into the MD algorithm itself. Simulations such as ours invariably contain adjustable parameters associated with numerical aspects of the dynamics computations (e.g. integration timesteps, thermostats, barostats). Moreover, the interatomic forcefields are typically phenomenological, with the associated tuning constants determined by comparison with experiments and/or first principles quantum calculations. We expect that analysis of these contributions could be addressed by viewing distinct sets of parameters as different models and attempting to quantify a between-model uncertainty analogous to our $\tau_{\beta, i}$. While such tasks are important, they remain beyond the scope of this work. See, e.g. Refs. [8] and [9] for works that have considered related problems.

We also omit a quantification of the model discrepancy, i.e. the extent to which the simulations disagree with experiment. In general, this is a difficult problem to approach for crosslinked polymers because in applications, one usually wishes to characterize such uncertainties as a function of the system chemistry, which is not a continuous or even ordered quantity. We leave this problem for future work.

\subsection{Extensions}

Even though the pooling analysis does not explicitly distinguish between the different sources of uncertainty affecting $T_{\mathrm{g}}$, it nonetheless provides a rough estimate associated with a simulation or data pool of a given size. As such, pooling can be used to construct an adaptive algorithm for determining $T_{\mathrm{g}}$ to within a given level of certainty. Provided that the simulations appear to be near the thermodynamic limit, the algorithm works as follows: (i) generate $M$ realizations of a system and compute the uncertainty $s^{2}(M-1)$; (ii) if this uncertainty is larger than 
desired, simulate one additional realization and compute $s^{2}(M) ; 656$ (iii) repeat until $s^{2}(M+n)$ is small enough for some $n \geq-1.657$ Given that large-scale simulations can be costly, the algorithm658 that we propose is a lower cost alternative that can yield po- $^{-}{ }_{659}$ tentially comparable results based on inexpensive, small sim- ${ }_{660}$ ulations. Moreover, this algorithm can permit optimal use of resources since processors need not be tied up for weeks or $_{662}$ months simulating large systems whose uncertainties are far $_{663}$ less than what may be required.

The key ideas underlying our analyses can also be extended ${ }_{665}^{664}$ to other types of simulated measurements. In particular, our use ${ }_{666}$ of global fits is predicated on the notion that physical properties ${ }_{667}$ are typically extracted from the behavior of a simulated sys- ${ }_{668}$ tem, i.e., its change in response to an external stimulus. Thus, ${ }_{669}$ finding functions that have the same general behavior as the un- ${ }_{670}$ derlying data can provide a means for objectively determining ${ }_{671}$ whether the latter actually has the characteristic features that ${ }_{672}$ define the physical property. Given a regression model from 673 which one estimates a quantity of interest, it is straightforward ${ }_{674}$ to invoke a pooling analysis to determine if the convergence of $f_{675}$ this quantity is consistent with the thermodynamic limit. We are pursuing similar lines of analysis for other material properties ${ }^{676}$ of interest and will report on such results in the future.

Acknowledgements: PNP was funded through the Institute ${ }_{670}$ for Mathematics and its Applications (IMA) by a grant from 680 NIST to the IMA. The IMA is a National Science Foundation ${ }_{681}$ Math Institute funded under award DMS-0931945. Thanks to 682 The Boeing Company for providing computational resources to ${ }_{683}$ perform the molecular dynamics simulations.

\section{Appendix A. Density convergence criterion}

The convergence criterion for densities is defined as follows. ${ }^{68}$ Denote an interval of times between time $t_{j}$ and $t_{j+1}$ as $I_{j}:{ }^{688}$ $\left[t_{j}, t_{j+1}\right]$, and partition this interval into $N_{j}$ equal timesteps $t_{j, k}{ }^{689}$ We use $I_{j}$ to represent a single simulation whose duration is ${ }^{690}$ equal to the length of the interval; $t_{j, k}\left(1 \leq k \leq N_{j}\right)$ denote the ${ }^{691}$ discrete simulation timesteps within that interval.

For both the equilibration and density-averaging algorithms, we first run a single simulation and compute the running average density

$$
\bar{\rho}_{r}\left(t_{1, k}\right)=\frac{1}{k} \sum_{k^{\prime}=1}^{k} \rho\left(t_{1, k^{\prime}}\right),
$$

where $\rho\left(t_{1, k^{\prime}}\right)$ is the density output by the simulation at the $k^{\prime}$ th $^{694}$ step in the 1 st interval. Next, we compute the "variance" 695

$$
V_{j}=\frac{1}{N_{j}} \sum_{k=1}^{N_{j}}\left[\bar{\rho}_{r}\left(t_{j, k}\right)-\bar{\rho}_{r}\left(t_{j, N_{j}}\right)\right]^{2}
$$

for $j=1$. If $V_{1}$ is less than some user-defined threshold $V_{\text {target }}{ }^{699}$ then the algorithm stops. If, on the other hand, $V_{1}>V_{\text {target }}, w \mathrm{w}_{701}$ run additional simulations, computing

$$
\bar{\rho}_{r}\left(t_{j+1, k}\right)=\frac{\left[\sum_{j, k^{\prime}} \rho\left(t_{j, k^{\prime}}\right)\right]+\sum_{k^{\prime}=1}^{k} \rho\left(t_{j+1, k^{\prime}}\right)}{\left[\sum_{j} N_{j}\right]+k}
$$

until $V_{j}<V_{\text {target }}$. Note that we calculate the running average across the sum of intervals $\sum_{j} I_{j}$, whereas we only compute the variance $V_{j}$ for individual intervals $I_{j}$.

In general, the task of picking suitable interval lengths $I$ and convergence criteria $V_{\text {target }}$ is non-trivial. Degenerate cases illustrate potential problems. If $I$ is so small as to encompass only a few timesteps, the system will not evolve from its initial state, and the variances $V_{j}$ will be artificially small. Consequently, the algorithm will stop prematurely. In the other extreme, picking $I$ to be on the scale of nanoseconds (which is long for MD simulations) can lead one to do more dynamical averaging than may be necessary. Picking $V_{\text {target }}$ too large or small leads to similar problems. For condensed polymer systems with $O\left(10^{4}\right)$ atoms or fewer, we find that setting $I=20 \mathrm{ps}$ is a reasonable compromise for all temperatures between $100 \mathrm{~K}$ and $800 \mathrm{~K}$, provided that our timesteps are $1 \mathrm{fs}$. This yields $N_{j}=20,000$ for all $j$. For equilibration steps and density averaging steps, we set $V_{\text {target }}=10^{-3} \mathrm{~g}^{2} / \mathrm{cm}^{6}$ and $V_{\text {target }}=10^{-7} \mathrm{~g}^{2} / \mathrm{cm}^{6}$, respectively. The latter value suggests that simulated densities are stable to within roughly $10^{-3} \mathrm{~g} / \mathrm{cm}^{3}$.

We caution that this stopping algorithm can severely undersample metastable energy minima of a single system, especially when the characteristic time to leave the mimima is on the order of or greater than the simulation lengths $I_{j}$. We attempt to compensate for this problem by generating multiple realizations of the same chemistry in order to more fully explore its possible configurations.

It is also important to note that our convergence criterion does not guarantee that the system statistics are representative of realistic equilibrium distributions. Reference [37] provides an analysis for assessing the extent to which this latter criterion has been satisfied on MD timescales, and this approach can likely be incorporated into our simulation procedure. However, we do not explicitly quantify uncertainties due to lack of such convergence because MD cannot reach the timescales necessary to verify that the aforementioned analysis is applicable to crosslinked polymers in the glassy regime.

\section{Appendix B. Proposition I}

In this section, we explain why $\tilde{T}$ and $s_{T_{\mathrm{g}}}^{2}$ are reasonable estimates of $T_{\mathrm{g}}$ and the uncertainty associated with data pools when $\mathcal{H}$ is linear. This task amounts to showing that such quantities are sample-based (and therefore computable), unbiased estimators for $\bar{T}$ and $\operatorname{Var}[T(\alpha)]$, which cannot be exactly computed. In particular, we demonstrate that $f_{m}$ defined by Eq. (16) undoes double counting associated with using the same datasets in different $\alpha$. A key benefit of Eq. (15) is that we can use this formula to estimate the variance $\operatorname{Var}[T(\alpha)]$ even though we have not run simulations corresponding to the pool size $|\alpha|$.

Proposition I. Let $\sigma^{2}:=\operatorname{Var}\left[x_{i}\right]$ for $x_{i}$ independent and identically distributed (i.i.d.). Then $\mathbf{E}[\tilde{x}(m)]=\bar{x}$ (i.e., $\tilde{x}(m)$ is an 
unbiased estimator of $\bar{x}$ ) and

$$
\begin{aligned}
s_{x}^{2}(m) & :=\frac{1}{f_{m}\left|A_{m}\right|} \sum_{\alpha \in A_{m}}\left(x_{\alpha}-\tilde{x}\right)^{2} \\
f_{m} & :=1-\sum_{\alpha, \alpha^{\prime} \in A_{m}} \frac{\left|\alpha \cap \alpha^{\prime}\right|}{m\left|A_{m}\right|^{2}}
\end{aligned}
$$

is an unbiased estimator of $\operatorname{Var}\left[x_{\alpha}\right]=\sigma^{2} /|\alpha|=\sigma^{2} / m$.

We first postulate that $x_{i}$ are independent and identically dis-714 tributed (i.i.d.) random variables, $\mathbf{E}\left[x_{i}\right]=\bar{x}$ is the mean value 715 of $x_{i}$, and $\operatorname{Var}\left[x_{i}\right]=\sigma^{2}$. It is easy to show that $\mathbf{E}[\tilde{x}(m)]=\bar{x}$ and ${ }_{716}$

$$
\operatorname{Var}\left[\sum_{i \in \alpha} \frac{x_{i}}{|\alpha|}\right]=\operatorname{Var}\left[x_{\alpha}\right]=E\left[\left(x_{\alpha}-\bar{x}\right)^{2}\right]=\frac{\sigma^{2}}{m} \text {. }
$$

This latter quantity should represent the variance of a system size corresponding to a pool size of $|\alpha|$. Supposing that we have $\left|A_{m}\right|$ data pools whose size is $m$, we wish to formulate a numerical recipe for estimating the true variance $\sigma^{2} /|\alpha|$ given only the ${ }_{721}$ data that we have actually generated. A key property of this recipe is that on average, it must yield the true variance $\sigma^{2} /|\alpha| ;_{723}$ this is what it means for an estimator to be unbiased.

To proceed, we postulate that this estimator can be expressed as

$$
\begin{aligned}
s^{2}(m) & =\frac{C}{\left|A_{m}\right|} \sum_{\alpha \in A_{m}}\left(x_{\alpha}-\tilde{x}\right)^{2}, \\
\tilde{x}(m) & :=\frac{1}{\left|A_{m}\right|} \sum_{\alpha \in A_{m}} x_{\alpha}
\end{aligned}
$$

where $C$ is some constant to be determined. Taking the expectation of Eq. (B.4), we can enforce the unbiased requirement by setting

$$
\mathbf{E}\left[s^{2}(m)\right]=\frac{C}{\left|A_{m}\right|} \mathbf{E}\left[\sum_{\alpha \in A_{m}}\left(x_{\alpha}-\tilde{x}\right)^{2}\right]=\frac{\sigma^{2}}{m} .
$$

Adding and subtracting $\bar{x}$ to the term in parentheses and invoking Eq. (B.3), we find,

$$
\begin{aligned}
\mathbf{E}\left[s^{2}(m)\right] & =\frac{C}{\left|A_{m}\right|} \mathbf{E}\left[\sum_{\alpha \in A_{m}}\left(x_{\alpha}-\bar{x}\right)^{2}-(\tilde{x}-\bar{x})^{2}\right] \\
& =C\left\{\frac{\sigma^{2}}{m}-\mathbf{E}\left[(\tilde{x}-\bar{x})^{2}\right]\right\}
\end{aligned}
$$

In order to simplify the last term, invoke the definition $x_{\alpha}:=$ $\sum_{i \in \alpha} x_{i} /|\alpha|$ and note that $\mathbf{E}\left[x_{i} x_{j}\right]=\sigma^{2} \delta_{i, j}$ (where $\delta_{i, j}$ is the Kronecker delta) to yield

$$
\begin{aligned}
\mathbf{E}\left[(\tilde{x}-\bar{x})^{2}\right] & =\sum_{\substack{\alpha \in A_{m} \\
\alpha^{\prime} \in A_{m}}} \sum_{\substack{i \in \alpha \\
j \in \alpha^{\prime}}} \frac{\mathbf{E}\left[\left(x_{i}-\bar{x}\right)\left(x_{j}-\bar{x}\right)\right]}{\left|A_{m}\right|^{2} m^{2}} \\
& =\sum_{\substack{\alpha \in A_{m} \\
\alpha^{\prime} \in A_{m}}} \sum_{\substack{i \in \alpha \cap \alpha^{\prime}\\
}} \frac{\sigma^{2}}{\left|A_{m}\right|^{2} m^{2}} \\
& =\sum_{\substack{\alpha \in A_{m} \\
\alpha^{\prime} \in A_{m}}} \frac{\sigma^{2}\left|\alpha \cap \alpha^{\prime}\right|}{\left|A_{m}\right|^{2} m^{2}}
\end{aligned}
$$

Thus we find that

$$
\mathbf{E}\left[s^{2}(m)\right]=\frac{\sigma^{2}}{m} C\left\{1-\sum_{\substack{\alpha \in A_{m} \\ \alpha^{\prime} \in A_{m}}} \frac{\left|\alpha \cap \alpha^{\prime}\right|}{m\left|A_{m}\right|^{2}}\right\},
$$

so that $C=f_{m}^{-1}$ in order that $\mathbf{E}\left[s^{2}(m)\right]=\sigma^{2} / m$. Note that when the $\alpha$ are independent, the above formula implies that $C=$ $\left(1-1 /\left|A_{m}\right|\right)^{-1}$, which is a well known result in statistics. As an aside, it is possible to recast set intersections of the form $\left|\alpha \cap \alpha^{\prime}\right|$ into inner products in an $m$-dimensional space. While it is beyond the scope of this work, additional results concerning, e.g. $\operatorname{Var}\left[s^{2}(m)\right]$ can easily be derived from this perspective and linear algebra.

\section{Appendix C. Proposition II}

Here we demonstrate that $\tilde{T}(m)$ is constant and $s_{T_{g}}^{2} \propto 1 / m$ if $\mathcal{H}$ is linear and $A_{m}$ samples all combinations of datasets. Before proceeding to the technical details, we propose a simple interpretation of this statement: exhaustive pooling entails uniform reuse of datasets, resulting in no new information about the sample mean and variance. In retrospect, the result can be anticipated, although its proof is, to our understanding, nontrivial.

Proposition II. Let $\hat{\sigma}_{x}^{2}$ be the sample variance of i.i.d. $x_{i}$, and assume that $A_{m}$ contains all combinations of data pools whose size $|\alpha|=m$. Then

$$
\begin{aligned}
\tilde{x}(m) & =\tilde{x}(1) \\
s_{x}^{2}(m) & =\hat{\sigma}_{x}^{2} / m
\end{aligned}
$$

holds identically.

Corollary Let $\mathcal{F}(x)$ be a function of $x$ and define $z_{i}=\mathcal{F}\left(x_{i}\right)$. If either

$$
\begin{aligned}
& \text { (1) }: \mathcal{F}(\tilde{x}(m)) \neq \sum_{i} z_{i} / M \text { or } \\
& \text { (2) }: s_{z}^{2}(m) \neq \hat{\sigma}_{z}^{2} / m
\end{aligned}
$$

then $\mathcal{F}(x)$ is a non-linear function of $x$.

It is straightforward to show that Eq. (C.1) holds because the $x_{i}$ are sampled symmetrically. To show Eq. (C.2), let $x_{i}$ be i.i.d. as before, and assume that $\tilde{\sigma}^{2}=s^{2}(1)$ applied to the realizations of $x$. Assume that $A_{m}$ contains all combinations of pools of size $m$, so that $\left|A_{m}\right|=\left(\begin{array}{c}M \\ m\end{array}\right)$. It is straightforward to show that, in this case, $\left|A_{m}\right| f_{m}=\left(\begin{array}{c}M \\ m\end{array}\right)(1-m / M)=\left(\begin{array}{c}M-1 \\ m\end{array}\right)$. Note also that $\tilde{x}=M^{-1} \sum_{i=1}^{M} x_{i}$, irrespective of the value of $m$. By definition

$$
s^{2}(m)=\frac{m^{-2}}{\left|A_{m}\right|(1-m / M)} \sum_{\alpha \in A_{m}} \sum_{\substack{i \in \alpha \\ j \in \alpha}}\left(x_{i}-\tilde{x}\right)\left(x_{j}-\tilde{x}\right) .
$$

Fix $j$ and note that (viewing sums from right to left) summing over every $i \in \alpha$ and then summing over $\alpha$ is equivalent to 
summing over every $\alpha: i \in \alpha$ and then summing over all $i$. That is

$$
\sum_{\alpha \in A_{m}} \sum_{i \in \alpha}\left(x_{i}-\tilde{x}\right)\left(x_{j}-\tilde{x}\right)=\sum_{i=1}^{M} \sum_{\substack{\alpha: i \in \alpha \\ \alpha \in A_{m}}}\left(x_{i}-\tilde{x}\right)\left(x_{j}-\tilde{x}\right) .
$$

Likewise, we can repeat this argument with $j$ to find that

$$
\begin{aligned}
\sum_{i=1}^{M} & \sum_{\substack{\alpha: i \in \alpha \\
\alpha \in A_{m}}} \sum_{j \in \alpha}\left(x_{i}-\tilde{x}\right)\left(x_{j}-\tilde{x}\right) \\
& =\sum_{i=1}^{M} \sum_{j=1}^{M} \sum_{\substack{\alpha: i, j \in \alpha \\
\alpha \in A_{m}}}\left(x_{i}-\tilde{x}\right)\left(x_{j}-\tilde{x}\right) .
\end{aligned}
$$

Hence, the question of computing the triple sum above amounts to understanding how many times a given pair of indices $i, j$ appears in the set $A_{m}$. To facilitate this, we use the decomposition

$$
\sum_{i=1}^{M} \sum_{j=1}^{M} \sum_{\substack{\alpha: i, j \in \alpha \\ \alpha \in A_{m}}}=\sum_{\substack{i=1 \\(i=j)}}^{M} \sum_{\substack{\alpha: i \in \alpha \\ \alpha \in A_{m}}}+\sum_{i=1}^{M} \sum_{\substack{j=1 \\ j \neq i}}^{M} \sum_{\substack{\alpha: i, j \in \alpha \\ \alpha \in A_{m}}}
$$

which implies

$$
\begin{aligned}
& \sum_{i=1}^{M} \sum_{j=1}^{M} \sum_{\substack{\alpha: i, j \in \alpha \\
\alpha \in A_{m}}}\left(x_{i}-\tilde{x}\right)\left(x_{j}-\tilde{x}\right)= \\
& \sum_{i=1}^{M} \sum_{\substack{\alpha: i \in \alpha \\
\alpha \in A_{m}}}\left(x_{i}-\tilde{x}\right)^{2}+\sum_{i=1}^{M} \sum_{\substack{j=1 \\
j \neq i}}^{M} \sum_{\substack{\alpha: i, j \in \alpha \\
\alpha \in A_{m}}}\left(x_{i}-\tilde{x}\right)\left(x_{j}-\tilde{x}\right)
\end{aligned}
$$

Denote these last two terms in Eq. (C.5) as Term I and Term II. ${ }_{\cdot 741}^{740}$

Consider first Term I. Note that when $\left|A_{m}\right|$ is maximal, we (742 $^{74}$ find $i \in \alpha$ a fraction $m / M=|\alpha| / M$ number of times, and hence ${ }^{743}$ $\left(x_{i}-\tilde{x}\right)^{2}$ should occur a total of $\frac{m}{M}\left(\begin{array}{c}M \\ m\end{array}\right)=\left(\begin{array}{c}M-1 \\ m-1\end{array}\right)$ times in Term $\mathrm{I}_{{ }_{745}}^{744}$ Thus,

$$
\sum_{i=1}^{M} \sum_{\substack{\alpha: i \in \alpha \\
\alpha \in A_{m}}}\left(x_{i}-\tilde{x}\right)^{2}=\left(\begin{array}{c}
M-1 \\
m-1
\end{array}\right)(M-1) \tilde{\sigma}^{2}
$$

Term II is simplified in a similar way. Note that of the ${ }^{753}$ $\left(\begin{array}{l}M-1 \\ m-1\end{array}\right)$ number of times that $i \in \alpha$, the index $j$ only appears ${ }_{755}^{754}$ in $(m-1) /(M-1)$ fraction of those sets. So the total number ${ }_{756}$ of times that a given pair $\left(x_{i}-\tilde{x}\right)\left(x_{j}-\tilde{x}\right)$ appears in Term II is ${ }^{757}$ $\frac{(m-1)}{(M-1)}\left(\begin{array}{c}M-1 \\ m-1\end{array}\right)=\left(\begin{array}{c}M-2 \\ m-2\end{array}\right)$. Hence

$$
\begin{aligned}
& \sum_{i=1}^{M} \sum_{\substack{j=1 \\
j \neq i}}^{M} \sum_{\substack{\alpha: i, j \in \alpha \\
\alpha \in A_{m}}}\left(x_{i}-\tilde{x}\right)\left(x_{j}-\tilde{x}\right) \\
& \quad=\sum_{i=1}^{M} \sum_{\substack{j=1 \\
j \neq i}}^{M}\left(\begin{array}{l}
M-2 \\
m-2
\end{array}\right)\left(x_{i}-\tilde{x}\right)\left(x_{j}-\tilde{x}\right) .
\end{aligned}
$$

\section{References}

[1] Proceedings of the 3rd World Congress on Integrated Computational Materials Engineering (ICME), Wiley, 2015.

[2] S. Christensen, A. Browning, J. Gosse, Computational Methods for New Materials Development, American Institute of Aeronautics and Astronautics, 2012. doi:doi:10.2514/6.2012-1487.

[3] C. Li, A. Strachan, Molecular scale simulations on thermoset polymers: A review, Journal of Polymer Science Part B: Polymer Physics 53 (2) (2015) 103-122. doi:10.1002/polb.23489.

[4] Integrated Computational Materials Engineering: A Transformational Discipline for Improved Competitiveness and National Security, The National Academies Press, 2008.

[5] A. Dienstfrey, J. Phelan, Frederick R., S. Christensen, A. Strachan, F. Santosa, R. Boisvert, Uncertainty quantification in materials modeling, JOM 66 (7) (2014) 1342-1344. doi:10.1007/s11837-014-1049-1.

[6] E. Donth, The Glass Transition: Relaxation Dynamics in Liquids and Disordered Materials, Physics and astronomy online library, Springer, 2001.

[7] P. Angelikopoulos, C. Papadimitriou, P. Koumoutsakos, Bayesian uncertainty quantification and propagation in molecular dynamics simulations: A high performance computing framework, The Journal of Chemical Physics 137 (14) (2012) 144103. doi:http://dx.doi.org/10.1063/1.4757266.

[8] F. Rizzi, R. E. Jones, B. J. Debusschere, O. M. Knio, Uncertainty quantification in md simulations of concentration driven ionic flow through a silica nanopore. i. sensitivity to physical parameters of the pore, The Journal of Chemical Physics 138 (19) (2013) 194104. doi:http://dx.doi.org/10.1063/1.4804666.

[9] F. Rizzi, R. E. Jones, B. J. Debusschere, O. M. Knio, Uncertainty quantification in md simulations of concentration driven ionic flow through a silica nanopore. ii. uncertain potential parameters, The Journal of Chemical Physics 138 (19) (2013) 194105. doi:http://dx.doi.org/10.1063/1.4804669. 
[10] F. Rizzi, H. N. Najm, B. J. Debusschere, K. Sargsyan, M. Salloum,84 H. Adalsteinsson, O. M. Knio, Uncertainty quantification in md simu-842 lations. part i: Forward propagation, Multiscale Modeling \& Simulation843 10 (4) (2012) 1428-1459. arXiv:http://dx.doi.org/10.1137/110853169,844 doi: $10.1137 / 110853169$

[11] F. Rizzi, H. N. Najm, B. J. Debusschere, K. Sargsyan, M. Sal-846 loum, H. Adalsteinsson, O. M. Knio, Uncertainty quantification in847 md simulations. part ii: Bayesian inference of force-field parame-848 ters, Multiscale Modeling \& Simulation 10 (4) (2012) 1460-1492.849 arXiv:http://dx.doi.org/10.1137/110853170, doi:10.1137/110853170

[12] C. Li, G. A. Medvedev, E.-W. Lee, J. Kim, J. M. Caruthers, 85 A. Strachan, Molecular dynamics simulations and experimen-852 tal studies of the thermomechanical response of an epoxy853 thermoset polymer, Polymer 53 (19) (2012) 4222 - 4230.854 doi:http://dx.doi.org/10.1016/j.polymer.2012.07.026.

13] B. Koo, Y. Liu, J. Zou, A. Chattopadhyay, L. L. Dai, Study of glass transi-856 tion temperature $(\mathrm{tg})$ of novel stress-sensitive composites using molec-857 ular dynamic simulation, Modelling and Simulation in Materials Science858 and Engineering 22 (6) (2014) 065018

[14] V. Varshney, S. S. Patnaik, A. K. Roy, B. L. Farmer, A molecu- 860 lar dynamics study of epoxy-based networks: Cross-linking procedure 861 and prediction of molecular and material properties, Macromolecules 862 41 (18) (2008) 6837-6842. arXiv:http://dx.doi.org/10.1021/ma801153e, doi:10.1021/ma801153e.

[15] C. Li, A. Strachan, Molecular simulations of crosslinking process of thermosetting polymers, Polymer 51 (25) (2010) 6058 - 6070

[16] C. Li, A. Strachan, Molecular dynamics predictions of thermal and mechanical properties of thermoset polymer epon862/detda, Polymer 52 (13) (2011) 2920 - 2928. doi:http://dx.doi.org/10.1016/j.polymer.2011.04.041.

[17] H. B. Fan, M. M. Yuen, Material properties of the crosslinked epoxy resin compound predicted by molecular dynamics simulation, Polymer 48 (7) (2007) 2174 - 2178. doi:http://dx.doi.org/10.1016/j.polymer.2007.02.007.

[18] S. Yu, S. Yang, M. Cho, Multi-scale modeling of cross-linked epoxy nanocomposites, Polymer 50 (3) (2009) 945 - 952. doi:http://dx.doi.org/10.1016/j.polymer.2008.11.054.

[19] C. Li, E. Coons, A. Strachan, Material property prediction of thermoset polymers by molecular dynamics simulations, Acta Mechanica 225 (4-5) (2014) 1187-1196. doi:10.1007/s00707-013-1064-2.

[20] P.-H. Lin, R. Khare, Molecular simulation of cross-linked epoxy and epoxyposs nanocomposite, Macromolecules 42 (12) (2009) 4319-4327. arXiv:http://dx.doi.org/10.1021/ma9004007, doi:10.1021/ma9004007.

[21] N. J. Soni, P.-H. Lin, R. Khare, Effect of cross-linker length on the thermal and volumetric properties of cross-linked epoxy networks: A molecular simulation study, Polymer 53 (4) (2012) 1015 - 1019. doi:http://dx.doi.org/10.1016/j.polymer.2011.12.051.

[22] T. W. Sirk, K. S. Khare, M. Karim, J. L. Lenhart, J. W. Andzelm, G. B. McKenna, R. Khare, High strain rate mechanical properties of a crosslinked epoxy across the glass transition, Polymer 54 (26) (2013) 7048 7057. doi:http://dx.doi.org/10.1016/j.polymer.2013.10.051.

[23] Materials Studio, version 7.1, BIOVIA, San Diego, California, 2014.

[24] H. C. Andersen, Molecular dynamics simulations at constant pressure and/or temperature, The Journal of Chemical Physics 72 (4).

[25] M. Parrinello, A. Rahman, Polymorphic transitions in single crystals: A new molecular dynamics method, Journal of Applied Physics 52 (12)

[26] MATLAB, version 8.1.0 (R2013a), The MathWorks Inc., Natick, Massachusetts, 2013.

[27] D. G. Watts, D. W. Bacon, Using an hyperbola as a transition model to fit two-regime straight-line data, Technometrics 16 (3) (1974) 369-373. arXiv:http://amstat.tandfonline.com/doi/pdf/10.1080/00401706.1974.10489205, doi:10.1080/00401706.1974.10489205.

[28] D. Griffiths, A. Miller, Hyperbolic regression - a model based on two-phase piecewise linear regression with a smooth transition between regimes, Communications in Statistics 2 (6) (1973) 561-569. arXiv:http://dx.doi.org/10.1080/03610927308827098, doi:10.1080/03610927308827098.

[29] J. Cologne, R. Sposto, Smooth piecewise linear regression splines with hyperbolic covariates, Journal of Applied Statistics 21 (4) (1994) 221233. arXiv:http://www.tandfonline.com/doi/pdf/10.1080/757583867, doi: $10.1080 / 757583867$.
[30] R. Pathria, Chapter 3 - The Canonical Ensemble, in: R. Pathria (Ed.), Statistical Mechanics, second edition Edition, Butterworth-Heinemann, Oxford, 1996, pp. 43 - 89. doi:http://dx.doi.org/10.1016/B978-0750624695/50005-0.

[31] JCGM/Working Group 1, Evaluation of measurement data-guide to the expression of uncertainty in measurement, Tech. Rep. JCGM 100:2008, Joint Commitee for Guides in Metrology (Sep. 2008).

[32] T. Hesterberg, Bootstrap, Wiley Interdisciplinary Reviews: Computational Statistics 3 (6) (2011) 497-526. doi:10.1002/wics.182.

[33] A. L. Rukhin, Weighted means statistics in interlaboratory studies, Metrologia 46 (3) (2009) 323.

[34] M. H. Quenouille, Notes on bias in estimation, Biometrika 43 (3/4) (1956) pp. 353-360.

[35] E. Jaramillo, N. Wilson, S. Christensen, J. Gosse, A. Strachan, Energy-based yield criterion for pmma from large-scale molecular dynamics simulations, Phys. Rev. B 85 (2012) 024114. doi:10.1103/PhysRevB.85.024114.

[36] G. H. Fredrickson, The equilibrium theory of inhomogeneous polymers, Clarendon, 2006.

[37] M. R. Shirts, Simple quantitative tests to validate sampling from thermodynamic ensembles, Journal of Chemical Theory and Computation 9 (2) (2013) 909-926, pMID: 26588735. doi:10.1021/ct300688p. 